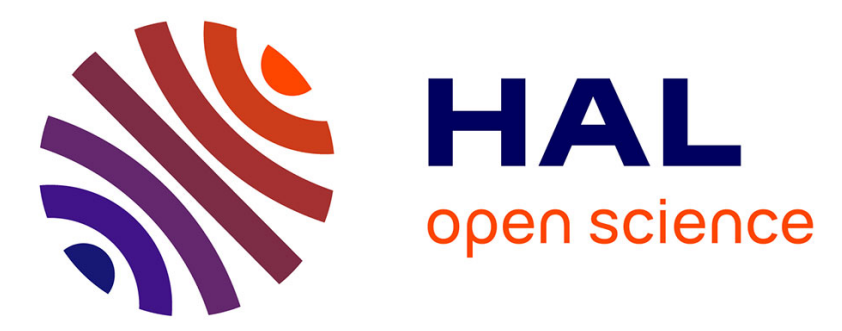

\title{
Remarks on the Trotter-Kato Product Formula for Unitary Groups
}

Pavel Exner, Hagen Neidhardt, Valentin A. Zagrebnov

\section{To cite this version:}

Pavel Exner, Hagen Neidhardt, Valentin A. Zagrebnov. Remarks on the Trotter-Kato Product Formula for Unitary Groups. Integral Equations and Operator Theory, 2011, 69 (4), pp.451-478. 10.1007/s00020-011-1867-2 . hal-00968609

\section{HAL Id: hal-00968609 https://hal.science/hal-00968609}

Submitted on 2 Apr 2014

HAL is a multi-disciplinary open access archive for the deposit and dissemination of scientific research documents, whether they are published or not. The documents may come from teaching and research institutions in France or abroad, or from public or private research centers.
L'archive ouverte pluridisciplinaire HAL, est destinée au dépôt et à la diffusion de documents scientifiques de niveau recherche, publiés ou non, émanant des établissements d'enseignement et de recherche français ou étrangers, des laboratoires publics ou privés. 


\title{
Remarks on the Trotter-Kato product formula for unitary groups
}

\author{
Pavel Exner, Hagen Neidhardt and Valentin A. Zagrebnov
}

Dedicated to our friend Takashi Ichinose in occasion of his 70th birthday

\begin{abstract}
Let $A$ and $B$ be non-negative self-adjoint operators in a separable Hilbert space such that their form sum $C$ is densely defined. It is shown that the Trotter product formula holds for imaginary parameter values in the $L^{2}$-norm, that is, one has

$$
\lim _{n \rightarrow+\infty} \int_{-T}^{T}\left\|\left(e^{-i t A / n} e^{-i t B / n}\right)^{n} h-e^{-i t C} h\right\|^{2} d t=0
$$

for each element $h$ of the Hilbert space and any $T>0$. This result is extended to the class of holomorphic Kato functions, to which the exponential function belongs. Moreover, for a class of admissible functions: $\phi(\cdot), \psi(\cdot): \mathbb{R}_{+} \longrightarrow \mathbb{C}$, where $\mathbb{R}_{+}:=[0, \infty)$, satisfying in addition $\Re \mathrm{e}(\phi(y)) \geq 0$, ऽm $(\phi(y) \leq 0$ and $\Im \mathrm{m}(\psi(y)) \leq 0$ for $y \in \mathbb{R}_{+}$, we prove that

$$
\text { s- } \lim _{n \rightarrow \infty}(\phi(t A / n) \psi(t B / n))^{n}=e^{-i t C}
$$

holds true uniformly on $[0, T] \ni t$ for any $T>0$.
\end{abstract}

Mathematics Subject Classification (2000). Primary 47A55, 47D03, 81Q30; Secondary 47B25.

Keywords. Trotter product formula, Trotter-Kato product formula, unitary groups, Feynman path integrals, holomorphic Kato functions, admissible functions.

\section{Introduction}

We open the present paper with a short survey of the main results on the TrotterKato product formula for imaginary times, and reformulate some of them in a form suitable for further generalizations. This allows us to extend the $L^{2}$-convergence of the imaginary-time Trotter product formula to holomorphic Kato functions. Using 
the concept of admissible functions introduced in [6] we prove this result also for the Trotter-Kato product formula.

It is a longtime open problem to prove that for non-negative self-adjoint operators $A$ and $B$ in a separable Hilbert space $\mathfrak{H}$ the strongly convergent Trotter product formula

$$
\text { S- } \lim _{n \rightarrow \infty}\left(e^{-i t A / n} e^{-i t B / n}\right)^{n}=e^{-i t C}
$$

holds uniformly in $t \in[0, T]$ for any $T>0$, where $C$ is the form sum of $A$ and $B$, cf. [13, Problem 11.3.9]. Apart from a pure mathematical interest such a product formula is tightly related to certain physical problems. In particular, the Trotter formula provides a natural way to define Feynman path integrals [4, 13]. Note that extensions of such a definition beyond the essentially self-adjoint case allows one to treat in this way Schrödinger operators for a much wider class of potentials.

In order to put our message into a proper context we recall first some known results relevant for our presentation. Let $-A$ and $-B$ be two generators of contraction semigroups in the Banach space $\mathfrak{X}$. In the seminal paper [22] Trotter proved that if the operator $-C$, where $C:=\overline{A+B}$, is a generator of a contraction semigroup on $\mathfrak{X}$, then the formula

$$
e^{-t C}=\mathrm{s}-\lim _{n \rightarrow \infty}\left(e^{-t A / n} e^{-t B / n}\right)^{n},
$$

holds for all $t \in[0, T]$ and any $T>0$. The formula is usually called Trotter, or Lie-Trotter product formula. The result was generalized by Chernoff in [2] to Banach spaces $\mathfrak{X}$ in the following form: Let $F(\cdot): \mathbb{R}_{+} \longrightarrow \mathfrak{B}(\mathfrak{X})$ be a strongly continuous operator-valued family of contractions such that $F(0)=I$ and the strong derivative $F^{\prime}(+0)$ exists being a densely defined operator in $\mathfrak{X}$. If $-C$, $C:=-\overline{F^{\prime}(+0)}$, is a generator of a $C_{0}$-contraction semigroup, then the generalized Lie-Trotter product formula

$$
e^{-t C}=\mathrm{s}-\lim _{n \rightarrow \infty} F(t / n)^{n},
$$

holds for $t \geq 0$. In [3, Theorem 3.1] it was shown that the strong convergence in the last formula is in fact uniform in $t \in[0, T]$ for any $T>0$.

Moreover, in [3, Theorem 1.1] this result was generalized as follows: Let $F(\cdot)$ : $\mathbb{R}_{+} \longrightarrow \mathfrak{B}(\mathfrak{X})$, where $\mathbb{R}_{+}=[0, \infty)$, be a family of linear contractions on a Banach space $\mathfrak{X}$. Then the generalized Lie-Trotter product formula (1.3) holds uniformly in $t \in[0, T]$ for any $T>0$ if and only if there is a $\lambda>0$ such that

$$
(\lambda+C)^{-1}=\text { s- } \lim _{\tau \rightarrow+0}\left(\lambda+S_{\tau}\right)^{-1},
$$

where

$$
S_{\tau}:=\frac{I-F(\tau)}{\tau}, \quad \tau>0 .
$$

Using these results, Kato [14] was able to prove the following claim: let $A$ and $B$ be two non-negative self-adjoint operators in a separable Hilbert space $\mathfrak{H}$. Assume 
that the intersection $\operatorname{dom}\left(A^{1 / 2}\right) \cap \operatorname{dom}\left(B^{1 / 2}\right)$ is dense in $\mathfrak{H}$. If $C:=A+B$ is the form sum of the operators $A$ and $B$, then Lie-Trotter product formula,

$$
e^{-t C}=\mathrm{s}-\lim _{n \rightarrow \infty}\left(e^{-t A / n} e^{-t B / n}\right)^{n},
$$

holds uniformly in $t \in[0, T]$ for any $T>0$. In fact, the Lie-Trotter formula was extended by Kato to more general products of the form $(f(t A / n) g(t B / n))^{n}$, where $f$ (and similarly $g$ ) is a real valued Borel measurable function $f(\cdot): \mathbb{R}_{+} \longrightarrow \mathbb{R}_{+}$ obeying $0 \leq f(t) \leq 1, f(0)=1$ and $f^{\prime}(+0)=-1$, which we call Kato functions in the following. Usually the product formulæ of that type are known under the name Lie-Trotter-Kato or Trotter-Kato.

It is a longstanding open question whether the Lie-Trotter product formula (1.4) remains valid for imaginary times $t$ under the same assumptions which justify the formula (1.2), see [3, Remark p. 91], [9], [10] and [20]. Note that if $A$ and $B$ are non-negative self-adjoint operators in $\mathfrak{H}$ and the limit in the left-hand side of (1.1) exists for all $t \in \mathbb{R}$, then $\operatorname{dom}\left(A^{1 / 2}\right) \cap \operatorname{dom}\left(B^{1 / 2}\right)$ is dense in $\mathfrak{H}$, see [13, Proposition 11.7.3]. Hence, we assume in the following that $\operatorname{dom}\left(A^{1 / 2}\right) \cap \operatorname{dom}\left(B^{1 / 2}\right)$ is dense in $\mathfrak{H}$. Furthermore, applying Trotter's result [22] one immediately gets that formula (1.1) is valid if the operator $C:=\overline{A+B}$ is self-adjoint. However, if $A+B$ is not essentially self-adjoint, then all attempts to verify the Lie-Trotter product formula (1.1) for imaginary times have failed so far. A somewhat weaker result is proved in [17, Proposition 3.2], see also [13, Proposition 11.7.4]. It was shown there that

$$
\text { s- } \lim _{n \rightarrow \infty} \int_{\mathbb{R}} \varphi(t)\left(e^{-i t A / n} e^{-i t B / n}\right)^{n} d t=\int_{\mathbb{R}} \varphi(t) e^{-i t C} d t, \quad C:=A \dot{+} B,
$$

holds for all $\varphi \in L^{1}(\mathbb{R})$.

In [10] Ichinose proposed a modified Trotter-type product formula. He proved in that paper that

$$
\begin{aligned}
e^{-i t C}= & \mathrm{s}-\lim _{n \rightarrow \infty}\left\{\left(e ^ { - i t A / n } \left(E_{A}([0, n \delta / t])+e^{-i t a / n} E_{A}((n \delta / t, \infty)) \times\right.\right.\right. \\
& \left(e^{-i t B / n}\left(E_{B}([0, n \delta / t])+e^{-i t b / n} E_{B}((n \delta / t, \infty))\right\}^{n}, \quad t \geq 0,\right.
\end{aligned}
$$

where $E_{A}(\cdot)$ and $E_{B}(\cdot)$ denote the spectral measures of the operators $A$ and $B$, respectively, and $a \geq 0, b \geq 0,0<\delta<\pi / 2$. If one introduces the functions

$$
f(\lambda):=e^{-i \lambda} \chi_{[0, \delta]}(\lambda)+\chi_{(\delta, \infty)}(\lambda), \quad \lambda \geq 0,
$$

then the result of [10] for $a=b=0$ acquires the form

$$
\text { s- } \lim _{n \rightarrow \infty}(f(t A / n) f(t B / n))^{n}=e^{-i t C}
$$

for any $t \geq 0$. Notice that the above function $f(\lambda)$ is admissible in the sense of [6], i.e.

$$
|f(x)| \leq 1, \quad x \in[0, \infty), \quad f(0)=1, \quad \text { and } \quad f^{\prime}(+0)=-i,
$$

and satisfies in addition the conditions $\Re \mathrm{e}(f(x)) \geq 0$ and $\Im \mathrm{m}(f(x)) \leq 0, x \in \mathbb{R}_{+}$. 
In [10, Section 3] this result was generalized to functions $\zeta(t, \lambda)$ from a class denoted as $\mathcal{F}_{\nu, \mu}(\tau, \gamma, \varepsilon), 0<\tau \leq \infty, 0<\mu<\nu \leq 1, \gamma \in \mathbb{R}$ and $\varepsilon= \pm 1$, defined in a slightly cumbersome way.

Consider a particular case. Let $f$ be an admissible function. Choosing $\gamma=0$ and $\varepsilon=-1$ one can verify that $\zeta(t, \lambda):=f(t \lambda) \in \mathcal{F}_{\nu, \mu}(\tau, 0,-1)$ if and only if $f(\cdot)$ is continuous,

$$
\Im \mathrm{m}(f(x)) \leq 0 \quad \text { and } \quad|1-f(x)| \leq \min \left\{2 \mu, \frac{1}{\nu}|\Im \mathrm{m}(f(x))|\right\}, \quad x \in \mathbb{R}_{+} .
$$

In particular, there exists a $\delta>0$ such that the conditions (1.9) are satisfied for

which yields

$$
f(x)=\frac{1}{1+i x} \chi_{[0, \delta]}(x)+\chi_{(\delta, \infty)}(x), \quad x \geq 0,
$$

$$
e^{-i t C}=\mathrm{s}-\lim _{n \rightarrow \infty}(f(t A / n) f(t B / n))^{n}, \quad t \geq 0 .
$$

In [15], see also [18] or [13, Corollary 11.3.5], Lapidus showed a slightly stronger result, namely that

$$
e^{-i t C}=\mathrm{s}-\lim _{n \rightarrow \infty}\left((I+i t A / n)^{-1}(I+i t B / n)^{-1}\right)^{n}
$$

holds uniformly in $t \in[0, T], T>0$.

Averaging formulas were proposed in [16] for real times for the cases of linear and non-linear semigroups. It was Cachia who for the first time linked the imaginary-time averaging formulas to the $L^{2}$-convergence. In [1] he proved that

$$
\lim _{n \rightarrow \infty} \int_{0}^{T}\left\|\left(\frac{e^{-2 i t A / n}+e^{-2 i t B / n}}{2}\right)^{n} h-e^{-i t C} h\right\|^{2} d t=0
$$

holds for any $h \in \mathfrak{H}$ and $T>0$. In fact, the notion of holomorphic Kato functions also appeared for the first time in [1]. A Kato function $f(\cdot)$ is called holomorphic, if it admits a holomorphic extention to the right complex half-plane, $\mathbb{C}_{\text {right }}:=\{z \in$ $\mathbb{C}: \Re \mathrm{e}(z)>0\}$, such that $|f(z)| \leq 1, z \in \mathbb{C}_{\text {right }}$. For holomorphic Kato functions the limit $f(i y):=\lim _{\epsilon \rightarrow+0} f(\epsilon+i y)$ exist for a.e. $y \in \mathbb{R}$. In the following we are going to show that there is a Borel measurable function $\widetilde{f}(\cdot): i \mathbb{R} \longrightarrow \mathbb{C}$ satisfying $|\widetilde{f}(i y)| \leq 1, y \in \mathbb{R}$, such that $f(i y)=\widetilde{f}(i y)$ for a.e. $y \in \mathbb{R}$, cf. Lemma 3.2. Since the $\widetilde{f}(\cdot)$ is Borel measurable the expression $\widetilde{f}(i s A)$ is well defined by the functional calculus for any $s \in \mathbb{R}$. Moreover, one has $\|\tilde{f}(i s A)\| \leq 1$ for $s \in \mathbb{R}$. It was shown in [1] that if $f$ and $g$ are holomorphic Kato functions, then

$$
\lim _{n \rightarrow \infty} \int_{0}^{T}\left\|\left(\frac{\tilde{f}(2 i t A / n)+\widetilde{g}(2 i t B / n)}{2}\right)^{n} h-e^{-i t C} h\right\|^{2} d t=0
$$

holds for any $h \in \mathfrak{H}$ and $T>0$.

Before we close this introductory survey, let us mention another family of related results. Note that the paper [1] was inspired, in fact, by results obtained by Ichinose and by one of us in [5]. This article was devoted to the so-called 
Zeno product formula, which can be regarded as a kind of degenerated Lie-Trotter product formula. In this formula one replaces the unitary factor $e^{-i t A}$ by an orthogonal projection onto some closed subspace $\mathfrak{h} \subseteq \mathfrak{H}$ and defines $C$ as the self-adjoint operator associated with the quadratic form $\mathfrak{k}(h, k):=(\sqrt{B} h, \sqrt{B} k)$, $h, k \in \operatorname{dom}(\mathfrak{k}):=\operatorname{dom}(\sqrt{B}) \cap \mathfrak{h}$, where it is assumed that $\operatorname{dom}(\mathfrak{k})$ is dense in $\mathfrak{h}$. It was proved in $[5]$ that

$$
\lim _{n \rightarrow \infty} \int_{0}^{T}\left\|\left(P e^{-i t B / n} P\right)^{n} h-e^{-i t C} h\right\| d t=0
$$

holds for any $h \in \mathfrak{h}$ and $T>0$, where $P$ is the orthogonal projection from $\mathfrak{H}$ onto $\mathfrak{h}$. Subsequently, an attempt was made in [6] to replace the strong $L^{2}$-topology of [5] by the usual strong topology of $\mathfrak{H}$. For admissible functions $\phi$ satisfying $\Im \mathrm{m}(\phi(x)) \leq 0, x \in \mathbb{R}_{+}$, it was shown in $[6]$ that

$$
e^{-i t C}=\mathrm{s}-\lim _{n \rightarrow \infty}(P \phi(t B / n) P)^{n},
$$

holds uniformly in $t \in[0, T]$ for any $T>0$. We would like to stress that the function $\phi(x)=e^{-i x}, x \in \mathbb{R}_{+}$, is admissible but does not satisfy the condition $\Im m\left(e^{-i x}\right) \leq 0$ for $x \in \mathbb{R}_{+}$, thus the question about convergence of the Zeno product formula in the strong topology of $\mathfrak{H}$ remains open.

Our present paper is organized as follows. In Section 2 we show that the Trotter product formula makes sense in $L^{2}$-topology, that is, it holds

$$
\lim _{n \rightarrow \infty} \int_{-T}^{T}\left\|\left(e^{-i t A / n} e^{-i t B / n}\right)^{n} h-e^{-i t C} h\right\|^{2} d t=0
$$

for $h \in \mathfrak{H}$ and any $T>0$ without any additional assumptions, cf. Theorem 2.2. This observation follows directly from the Lapidus result (1.5). Of course, it does not solve under our hypotheses the strong convergence problem of (1.1). Nevertheless, (1.11) implies the existence of a subsequence $n_{k}$ such that one has pointwise (i.e., the strong) convergence along it for a.e. $t \in[-T, T]$. From the physical point of view our result seems to be quite satisfactory, see a discussion on that point in $[8$, Section 11].

Using the concept of the holomorphic Kato functions we prove the TrotterKato product formula in the $L^{2}$-topology in Section 3 , that is,

$$
\lim _{n \rightarrow \infty} \int_{-T}^{T}\left\|(\tilde{f}(i t A / n) \widetilde{g}(i t B / n))^{n} h-e^{-i t C} h\right\|^{2} d t=0
$$

for $h \in \mathfrak{H}$ and any $T>0$, where $f, g$ are holomorphic Kato functions and $\widetilde{f}, \widetilde{g}$ are Borel measurable extensions of $f$ and $g$ on the imaginary axis, see Lemma 3.2 and Theorem 3.3. Moreover, we propose a characterization of the class of holomorphic Kato functions.

Finally, in Section 4 we give a generalization of the results due to Ichinose [10], to the class of admissible functions defined above. We show that

$$
\text { s- } \lim _{n \rightarrow \infty}(\phi(t A / n) \psi(t B / n))^{n}=e^{-i t C},
$$


where $\phi$ and $\psi$ are admissible functions such that $\Re \mathrm{e}(\phi(y)) \geq 0$, ऽm $(\phi(y) \leq 0$ and $\Im \mathrm{m}(\psi(y)) \leq 0$ for $y \in \mathbb{R}_{+}$, cf. Theorem 4.7. Choosing $\phi(y)=\psi(y)=(1+i y)^{-1}$, $y \in \mathbb{R}$, one recovers Lapidus' result (1.10), see [18] and [13, Corollary 11.3.5]. Moreover, it turns out that admissible functions can be always slightly modified so that the Trotter-Kato product formula is valid, see Corollary 4.9. In particular, it follows from Corollary 4.9 that the modified Trotter product formula,

$$
\text { s- } \lim _{n \rightarrow \infty}\left(e^{-i t A / n} E_{A}([0, \pi n / 2 t]) e^{-i t B / n} E_{B}([0, \pi n / 2 t])\right)^{n}=e^{-i t C},
$$

holds uniformly in $t \in[0, T], T>0$, cf. (1.7) and (1.8). Notice that (1.14) is similar to $(1.6)$.

\section{Lapidus' results revisited}

We start by proving the following important technical lemma.

Lemma 2.1. Let $\left\{F_{n}(\cdot)\right\}_{n \in \mathbb{N}}$ be a family of measurable operator-valued functions $F_{n}(\cdot): i \mathbb{R} \longrightarrow \mathcal{B}(\mathfrak{H})$ such that $\left\|F_{n}(i t)\right\| \leq 1$ holds for a.e. $t \in \mathbb{R}$. Furthermore, let $C$ be a densely defined self-adjoint operator. Then the following assertions are equivalent:

(i) For each $\varphi \in L^{1}(\mathbb{R})$ one has

$$
\text { W- } \lim _{n \rightarrow \infty} \int_{\mathbb{R}} \varphi(t) F_{n}(i t) d t=\int_{\mathbb{R}} \varphi(t) e^{-i t C} d t .
$$

(ii) For each $h \in \mathfrak{H}$ and $T>0$ it holds

$$
\lim _{n \rightarrow \infty} \int_{-T}^{T}\left\|F_{n}(i t) h-e^{-i t C} h\right\|^{2} d t=0 .
$$

(iii) For each $\varphi \in L^{1}(\mathbb{R})$ one has

$$
\text { s- } \lim _{n \rightarrow \infty} \int_{\mathbb{R}} \varphi(t) F_{n}(i t) d t=\int_{\mathbb{R}} \varphi(t) e^{-i t C} d t .
$$

Proof. (i) $\Longrightarrow$ (ii) Since

$$
\left\|F_{n}(i t) h-e^{-i t C} h\right\|^{2} \leq 2\|h\|^{2}-2 \Re \mathrm{e}\left(F_{n}(i t) h, e^{-i t C} h\right), \quad t \in \mathbb{R},
$$

and

$$
e^{-i t C} h=\sum_{k=0}^{\infty} \frac{(-i t)^{k}}{k !} C^{k} h, \quad t \in \mathbb{R},
$$

for $h \in E_{C}([a, b]) \mathfrak{H},-\infty<a<b<\infty$, we find

$$
\left\|F_{n}(i t) h-e^{-i t C} h\right\|^{2} \leq 2\|h\|^{2}-2 \Re \mathrm{e}\left(\sum_{k=0}^{\infty} \frac{i^{k} t^{k}}{k !}\left(F_{n}(i t) h, C^{k} h\right)\right)
$$


for a.e. $t \in \mathbb{R}$, which leads to

$$
\int_{-T}^{T}\left\|F_{n}(i t) h-e^{-i t C} h\right\|^{2} d t \leq 4 T\|h\|^{2}-2 \Re \mathrm{e}\left(\sum_{k=0}^{\infty} \frac{i^{k}}{k !} \int_{-T}^{T} t^{k}\left(F_{n}(i t)^{n} h, C^{k} h\right) d t\right)
$$

or

$$
\int_{-T}^{T}\left\|F_{n}(i t) h-e^{-i t C} h\right\|^{2} d t \leq 4 T\|h\|^{2}-2 \Re \mathrm{e}\left(\sum_{k=0}^{\infty} \frac{i^{k}}{k !}\left(\int_{-T}^{T} t^{k} F_{n}(i t) h d t, C^{k} h\right)\right)
$$

for $t \geq 0$. From (2.1) we get

$$
\lim _{n \rightarrow \infty}\left(\int_{-T}^{T} t^{k} F_{n}(i t) h, C^{k} h\right)=\left(\int_{-T}^{T} t^{k} e^{-i t C} h d t, C^{k} h\right) .
$$

Hence

$$
\int_{-T}^{T}\left\|F_{n}(i t) h-e^{-i t C} h\right\|^{2} d t \leq 4 T\|g\|^{2}-2 \Re \mathrm{e}\left(\sum_{k=0}^{\infty} \frac{i^{k}}{k !}\left(\int_{-T}^{T} t^{k} e^{-i t C} h d t, C^{k} h\right)\right) .
$$

Therefore

$$
\limsup _{n \rightarrow \infty} \int_{-T}^{T}\left\|F_{n}(i t) h-e^{-i t C} h\right\|^{2} d t \leq 4 T\|h\|^{2}-2 \Re e \int_{-T}^{T}\left(e^{-i t C} h, e^{-i t C} h\right) d t=0
$$

which proves (2.2).

(ii) $\Longrightarrow$ (iii) The following estimate holds:

$$
\left\|\int_{-T}^{T} \varphi(t)\left(F_{n}(i t)-e^{-i t C}\right) h d t\right\| \leq \int_{-T}^{T}|\varphi(t)|\left\|F_{n}(i t) h-e^{-i t C} h\right\| d t .
$$

From (1.11) we obtain the convergence in measure, that is, for each $\varepsilon>0$ one has

$$
\lim _{n \rightarrow \infty}\left|\left\{t \in[-T, T]:\left\|F_{n}(i t) h-e^{-i t C} h\right\| \geq \varepsilon\right\}\right|=0 .
$$

Setting $\Delta_{\varepsilon, n}:=\left\{t \in[-T, T]:\left\|F_{n}(i t) h-e^{-i t C} h\right\| \geq \varepsilon\right\}$ we find the estimate

$$
\begin{aligned}
& \left\|\int_{-T}^{T} \varphi(t)\left(F_{n}(i t)-e^{-i t C}\right) h d t\right\| \\
& \quad \leq \varepsilon \int_{[-T, T] \backslash \Delta_{\varepsilon, n}}|\varphi(t)| d t+2 \int_{\Delta_{\varepsilon, n}}|\varphi(t)| d t, \quad n \in \mathbb{N} .
\end{aligned}
$$

In view of (2.2) we obtain in the limit $n \rightarrow \infty$ the inequality

$$
\limsup _{n \rightarrow \infty}\left\|\int_{-T}^{T} \varphi(t)\left(F_{n}(i t)-e^{-i t C}\right) h d t\right\| \leq \varepsilon \int_{\mathbb{R}}|\varphi(t)| d t
$$

for any $\varepsilon>0$. Hence for any $\varepsilon$ small enough we have

$$
\limsup _{n \rightarrow \infty}\left\|\int_{\mathbb{R}} \varphi(t)\left(F_{n}(i t)-e^{-i t C}\right) h d t\right\| \leq \varepsilon \int_{\mathbb{R}}|\varphi(t)| d t+2 \int_{\mathbb{R} \backslash[-T, T]}|\varphi(t)| d t .
$$


Since $T$ can be chosen sufficiently large and $\varepsilon$ was arbitrary we get

which yields

$$
\limsup _{n \rightarrow \infty}\left\|\int_{\mathbb{R}} \varphi(t)\left(F_{n}(i t)-e^{-i t C}\right) h d t\right\|=0,
$$

$$
\text { s- } \lim _{n \rightarrow \infty} \int_{\mathbb{R}} \varphi(t) F_{n}(i t) h=\int_{\mathbb{R}} \varphi(t) e^{-i t C} h d t, \quad h \in \mathfrak{H} .
$$

(iii) $\Longrightarrow$ (i) Obviously (2.3) implies (2.1).

Lemma 2.1 allows us to reformulate the Lapidus result of [17, Proposition $3.2]$, mentioned as (1.5) above, in the following form:

Theorem 2.2. Let $A$ and $B$ two non-negative self-adjoint operators on the Hilbert space $\mathfrak{H}$. If the form sum $C:=A+B$ is densely defined, then (1.11) holds for any $h \in \mathfrak{H}$ and $T>0$.

Proof. We set

$$
F_{n}(i t):=\left(e^{-i t A / n} e^{-i t B / n}\right)^{n}, \quad n \in \mathbb{N}, \quad t \in \mathbb{R} .
$$

From [17, Proposition 3.2] we get (1.5), which yields (2.1). Applying now Lemma 2.1 we obtain (1.11).

We note that Theorem 2.2 partially solves the question posed in [13, Problem 11.3.9] by a slight change of topology. Indeed, from Theorem 2.2 we get that (1.1) holds in measure, that is, for any $\eta>0, h \in \mathfrak{H}$ and $T>0$ one has

$$
\lim _{n \rightarrow \infty}\left|\left\{t \in[-T, T]:\left\|\left(e^{-i t A / n} e^{-i t B / n}\right)^{n} h-e^{-i t C} h\right\| \geq \eta\right\}\right|=0,
$$

where $|\cdot|$ denotes the Lebesgue measure, while [13, Problem 11.3.9] requires a uniform convergence of $t \in[-T, T]$, i.e. for any $\eta>0, h \in \mathfrak{H}$ and $T>0$ one has

$$
\lim _{n \rightarrow \infty} \sup _{t \in[-T, T]}\left\|\left(e^{-t A / n} e^{-t B / n}\right)^{n} h-e^{-t C} h\right\|=0 .
$$

Notice that convergence in measure (2.5) takes place if and only if any subsequence of $\left\{\left(e^{-i t A / n} e^{-i t B / n}\right)^{n}\right\}_{n \in \mathbb{N}}$ contains a subsequence $\left\{\left(e^{-i t A / n_{k}} e^{-i t B / n_{k}}\right)^{n_{k}}\right\}_{k \in \mathbb{N}}$ which converges strongly almost everywhere to $e^{-i t C}$, i.e

$$
\mathrm{s}-\lim _{k \rightarrow \infty}\left(e^{-i t A / n_{k}} e^{-i t B / n_{k}}\right)^{n_{k}}=e^{-i t C}
$$

holds for a.e. $t \in[-T, T]$.

Remark 2.3. From the viewpoint of physical applications, the formula (1.11) allows us to extend the Trotter-type definition of Feynman integrals for Schrödinger operators to a wider class of potentials. Following [13, Definition 11.2.21] the Feynman integral $\mathcal{F}_{\mathrm{TP}}^{t}(V)$ associated with the potential $V$ is the strong operator limit

$$
\mathcal{F}_{\mathrm{TP}}^{t}(V):=\mathrm{s}-\lim _{n \rightarrow \infty}\left(e^{-i t H_{0} / n} e^{-i t V / n}\right)^{n}
$$


where $H_{0}:=-\frac{1}{2} \Delta$ and $-\Delta$ is the Laplacian operator in $L^{2}\left(\mathbb{R}^{d}\right)$ defined in the usual way. From [13, Corollary 11.2.22] one gets that the Feynman integral exists if $V: \mathbb{R}^{d} \longrightarrow \mathbb{R}$ is Lebesgue measurable, non-negative, and satisfies $V \in L_{\text {loc }}^{2}\left(\mathbb{R}^{d}\right)$.

With Theorem 2.2 in mind it is possible to extend the Trotter-type definition of Feynman integrals if one replaces the $L^{2}\left(\mathbb{R}^{d}\right)$-topology by the $L^{2}\left([-T, T] \times \mathbb{R}^{d}\right)$ topology. Indeed, let us define the generalized Feynman integral $\mathcal{F}_{\text {gTP }}^{t}(V)$ by

$$
\lim _{n \rightarrow \infty} \int_{-T}^{T}\left\|\left(e^{-i t H_{0} / n} e^{-i t V / n}\right)^{n} h-\mathcal{F}_{\mathrm{gTP}}^{t}(V) h\right\|^{2} d t=0
$$

for $h \in L^{2}\left(\mathbb{R}^{d}\right)$ and $T>0$. Obviously, the existence of $\mathcal{F}_{\mathrm{TP}}^{t}(V)$ yields the existence of $\mathcal{F}_{\text {gTP }}^{t}(V)$ while the converse is in general not true. By Theorem 2.2 one can immediately conclude that the generalized Feynman integral exists if $V: \mathbb{R}^{d} \longrightarrow \mathbb{R}$ is Lebesgue measurable, non-negative, and satisfies $V \in L_{\text {loc }}^{1}\left(\mathbb{R}^{d}\right)$. This substantially extends the class of admissible potentials. The same class of potentials is covered by the so-called modified Feynman integral $\mathcal{F}_{M}^{t}(V)$ defined by

$$
\mathcal{F}_{M}^{t}(V):=\mathrm{s}-\lim _{n \rightarrow \infty}\left(\left[I+i(t / n) H_{0}\right]^{-1}[I+i(t / n) V]^{-1}\right)^{n},
$$

see [13, Definition 11.4.4] and [13, Corollary 11.4.5]. However, in this case the exponents are replaced by resolvents which leads to loss of the typical structure of Feynman integrals and the related physical insights.

\section{Lapidus' result generalized}

The Lapidus result (1.5) relies on the so-called Vitali's classical theorem and the Vitali extended theorem, cf. [13, Theorem 11.7.1]. We reformulate them in application to our situation as follows:

Let $\Phi_{n}(z), n \in \mathbb{N}$ be a sequence of contractive holomorphic function in $\mathbb{C}_{\text {right }}$ which for $x \in \mathbb{R}_{+}$converges to a function $\Phi(x)$, that is, $\lim _{n \rightarrow \infty} \Phi_{n}(x)=\Phi(x)$ for $x \in \mathbb{R}_{+}$. Then $\Phi(x)$ admits a contractive holomorphic continuation $\Phi(z)$ to $\mathbb{C}_{\text {right }}$ such that $\Phi(z)=\lim _{n \rightarrow \infty} \Phi_{n}(z)$. Since $\Phi_{n}(z)$ and $\Phi(z)$ are contractive holomorphic functions the limits $\Phi_{n}(i y):=\lim _{\epsilon \rightarrow+0} \Phi_{n}(\epsilon+i y), n \in \mathbb{N}$, and $\Phi(i y):=$ $\lim _{\epsilon \rightarrow+0} \Phi(\epsilon+i y)$ exist for a.e. $y \in \mathbb{R}$. The Vitali extended theorem now yields that

$$
\lim _{n \rightarrow \infty} \int_{\mathbb{R}} \varphi(y) \Phi_{n}(i y) d y=\int_{\mathbb{R}} \varphi(y) \Phi(i y) d y
$$

for any $\varphi \in L^{1}(\mathbb{R})$. Notice that this conclusion cannot be deduced from Theorem 11.7.1 of [13], since it is required that the functions $\Phi_{n}(z)$ and $\Phi(z)$ must admit continuous extension to $\overline{C_{\text {right }}}$. However, applying Lemma 2 of [1], which is a slight generalization of Theorem 11.7.1 from [13], one gets that the conclusion holds.

Let us make precise the notion of holomorphic Kato functions (cf. Section 1) in the following way: 
Definition 3.1. Let $f(\cdot): \mathbb{R}_{+} \longrightarrow \mathbb{R}_{+}$be a Kato function. The function is called a holomorphic Kato function if $f(\cdot)$ admits a holomorphic continuation to $\mathbb{C}_{\text {right }}$ such that

$$
|f(z)| \leq 1, \quad z \in \mathbb{C}_{\text {right }} .
$$

Standard holomorphic Kato functions are $f_{k}(x):=(1+x / k)^{-k}, x \in \mathbb{R}_{+}$, and, of course, $f(x)=e^{-x}, x \in \mathbb{R}_{+}$. At the end of this section we give a description of holomorphic Kato functions and indicate some non-standard examples of holomorphic Kato functions. It turns out that for standard holomorphic functions the limit to the imaginary axis exists everywhere. This yields $f(t A)=\mathrm{s}-\lim _{\epsilon \rightarrow+0} f((\epsilon+i t) A)$ for any $t \geq 0$. However, if $f$ is a holomorphic Kato function, then in general the relation $f(i t A)=\mathrm{s}^{-\lim _{\epsilon \rightarrow+0}} f((\epsilon+i t) A)$ cannot be expected. Indeed, this is due the fact that the limit $f(i y)=\lim _{\epsilon \rightarrow+0} f(\epsilon+i y)$ exists only for a.e. $y \in \mathbb{R}$. Hence the limit function $f(i y)$ is not in general Borel measurable which makes it impossible to apply the functional calculus for self-adjoint operators. However, the limit function $f(i y)$, defined for all those $y \in \mathbb{R}$ for which the limit $f(i y)$ exists, admits an extension to the whole real axis which is Borel measurable.

Lemma 3.2. Let $f(\cdot): \mathbb{R}_{+} \longrightarrow \mathbb{R}_{+}$be a holomorphic Kato function. Then there is a Borel measurable function $\tilde{f}(\cdot): i \mathbb{R} \longrightarrow \mathbb{C}$ satisfying $|\widetilde{f}(i y)| \leq 1, y \in \mathbb{R}$, such that $\tilde{f}(i y)=\lim _{\epsilon \rightarrow+0} f(\epsilon+i y)$ for a.e. $y \in \mathbb{R}$.

Proof. We set $f_{R}(z):=\Re \mathrm{e}(f(z))$ and $f_{I}(z)=\Im \mathrm{m}(f(z)), z \in \mathbb{C}_{\text {right }}$. Since $|f(z)| \leq$ $1, z \in \mathbb{C}_{\text {right }}$, we find

$$
\left|f_{R}(z)\right|^{2}+\left|f_{I}(z)\right|^{2} \leq 1, \quad z \in \mathbb{C}_{\text {right }} .
$$

Further we set $f_{R}^{ \pm}(z):=\max \left\{0, \pm f_{R}(z)\right\} \geq 0$ and $f_{I}^{ \pm}(z):=\max \left\{0, \pm f_{I}(z)\right\} \geq 0$, $z \in \mathbb{C}_{\text {right }}$. Since the function $f(\cdot)$ is holomorphic the functions $f_{R}^{ \pm}(\cdot)$ and $f_{I}^{ \pm}(\cdot)$ are Borel measurable. Obviously, we have

$$
f(z)=f_{R}^{+}(z)-f_{R}^{-}(z)+i\left(f_{I}^{+}(z)-f_{I}^{-}(z)\right), \quad z \in \mathbb{C}_{\text {right }}
$$

and

$$
\left|f_{R}^{+}(z)\right|^{2}+\left|f_{R}^{-}(z)\right|^{2}+\left|f_{I}^{+}(z)\right|^{2}+\left|f_{I}^{-}(z)\right|^{2} \leq 1, \quad z \in \mathbb{C}_{\text {right }} .
$$

We set $\tilde{f}_{R}^{ \pm}(i y):=\liminf _{\epsilon \rightarrow+0} f_{R}^{ \pm}(\epsilon+i y)$ and $\tilde{f}_{I}^{ \pm}(i y):=\liminf _{\epsilon \rightarrow+0} f_{I}^{ \pm}(\epsilon+i y)$, $y \in \mathbb{R}$. Since $f_{R}^{ \pm}(z)$ and $f_{I}^{ \pm}(z), z \in \mathbb{C}_{\text {right }}$, are Borel measurable functions, the functions $\tilde{f}_{R}^{ \pm}(i y)$ and $\tilde{f}_{I}^{ \pm}(i y)$ are also Borel measurable. From

$$
\inf _{0<\eta \leq \epsilon} f_{R}^{ \pm}(\eta+i y) \leq f_{R}^{ \pm}(\epsilon+i y) \quad \text { and } \quad \inf _{0<\eta \leq \epsilon} f_{I}^{ \pm}(\eta+i y) \leq f_{I}^{ \pm}(\epsilon+i y), \quad y \in \mathbb{R},
$$

we find

$$
\begin{aligned}
& \sum_{j= \pm}\left(\left|\inf _{0<\eta \leq \epsilon} f_{R}^{j}(\eta+i y)\right|^{2}+\left|\inf _{0<\eta \leq \epsilon} f_{I}^{j}(\eta+i y)\right|^{2}\right) \\
& \quad \leq\left|f_{R}^{+}(\epsilon+i y)\right|^{2}+\left|f_{R}^{-}(\epsilon+i y)\right|^{2}+\left|f_{I}^{+}(\epsilon+i y)\right|^{2}+\left|f_{I}^{-}(\epsilon+i y)\right|^{2} \leq 1,
\end{aligned}
$$


$y \in \mathbb{R}$, which yields

$$
\left|\tilde{f}_{R}^{+}(i y)\right|^{2}+\left|\tilde{f}_{R}^{-}(i y)\right|^{2}+\left|\tilde{f}_{I}^{+}(i y)\right|^{2}+\left|\tilde{f}_{I}^{-}(i y)\right|^{2} \leq 1, \quad y \in \mathbb{R} .
$$

Setting

$$
\widetilde{f}(i y):=\widetilde{f}_{R}^{+}(i y)-\tilde{f}_{R}^{-}(i y)+i\left(\tilde{f}_{I}^{+}(i y)-\tilde{f}_{I}^{-}(i y)\right), \quad y \in \mathbb{R},
$$

we define a Borel measurable function. From

$$
\begin{aligned}
& |\widetilde{f}(i y)|^{2}=\left|\widetilde{f}_{R}^{+}(i y)-\widetilde{f}_{R}^{-}(i y)\right|^{2}+\left|\widetilde{f}_{I}^{+}(i y)-\widetilde{f}_{I}^{-}(i y)\right|^{2} \\
& \quad \leq\left|\widetilde{f}_{R}^{+}(i y)\right|^{2}+\left|\widetilde{f}_{R}^{-}(i y)\right|^{2}+\left|\widetilde{f}_{I}^{+}(i y)\right|^{2}+\left|\widetilde{f}_{I}^{-}(i y)\right|^{2} \leq 1, \quad y \in \mathbb{R} .
\end{aligned}
$$

The relation $\tilde{f}(i y)=\lim _{\epsilon \rightarrow+0} f(\epsilon+i y)$ for a.e. $y \in \mathbb{R}$ is obvious.

Since $|\tilde{f}(i y)| \leq 1, y \in \mathbb{R}$, the expression $\tilde{f}(i \tau A), \tau \in \mathbb{R}$, is well defined for any self-adjoint operator $A$.

Setting $F(z):=f(z A) g(z B), z \in \mathbb{C}_{\text {right }}$, it turns out that the operatorvalued family $F(z)$ is contractive and holomorphic, if $f$ and $g$ are holomorphic Kato functions.

Theorem 3.3. Let $A$ and $B$ two non-negative self-adjoint operators on the Hilbert space $\mathfrak{H}$. Assume that $C:=A+B$ is densely defined. If $f$ and $g$ are holomorphic Kato functions, then (1.12) holds for any $h \in \mathfrak{H}$ and $T>0$.

Proof. We set $F_{n}(z):=F(z / n)^{n}, z \in \overline{\mathbb{C}_{\text {right }}}, n \in \mathbb{N}$. We note that if $z=t \in \mathbb{R}_{+}$, then

$$
F_{n}(t):=(f(t A / n) g(t B / n))^{n}=\left(e^{-t A / n} e^{-t B / n}\right)^{n}, \quad t \in \mathbb{R}_{+}, \quad n \in \mathbb{N} .
$$

From [14] we find

for $t \in \mathbb{R}_{+}$which yields

$$
\text { S- } \lim _{n \rightarrow \infty} F_{n}(t)=e^{-t C}
$$

$$
\lim _{n \rightarrow \infty}\left(F_{n}(t) h, k\right)=\left(e^{-t C} h, k\right)
$$

for $t \in \mathbb{R}_{+}$and $h, k \in \mathfrak{H}$. Setting $\Phi_{n}(t):=\left(F_{n}(t) h, k\right), n \in \mathbb{N}$, and $\Phi(t)=$ $\left(e^{-t C} h, k\right), t \in \mathbb{R}_{+}$, by Vitali's classical theorem, see [13, Theorem 11.7.1.(i)], we find $\lim _{n \rightarrow \infty} \Phi_{n}(z)=\Phi(z), z \in \mathbb{C}_{\text {right }}$. Moreover, taking into account the extended Vitali theorem, see Lemma 2.1 of [1], we get

$$
\lim _{n \rightarrow \infty} \int_{\mathbb{R}} \varphi(t) \Phi_{n}(i t) d t=\int_{\mathbb{R}} \varphi(t) \Phi(i t) d t
$$

for all $\varphi \in L^{1}(\mathbb{R})$. The last relation yields

$$
\mathrm{w}-\lim _{n \rightarrow \infty} \int_{\mathbb{R}} \varphi(t) F_{n}(i t) d t=\int_{\mathbb{R}} \varphi(t) e^{-i t C} d t
$$

for all $\varphi \in L^{1}(\mathbb{R})$ where $F_{n}(i t):=\operatorname{s} \lim _{\epsilon \rightarrow+0} F_{n}(\epsilon+i t)$ for a.e. $t \in \mathbb{R}$, cf. [21, Section V.2]. Applying Lemma 2.1 we obtain

$$
\lim _{n \rightarrow \infty} \int_{-T}^{T}\left\|F_{n}(i t) h-e^{-i t C} h\right\|^{2} d t=0
$$


for $T>0$.

It remains to show that $F_{n}(i t)$ can be replaced by $(\widetilde{f}(i t A / n) \widetilde{g}(i t B / n))^{n}$ for each $n \in \mathbb{N}$. We find

$$
\begin{array}{rl}
\int_{-T}^{T} & d t\|f((\epsilon+i t) A / n) h-\tilde{f}(i t A / n) h\|^{2} \\
& =\int_{-T}^{T} d t \int_{[0, \infty)} d\left(E_{A}(\lambda) h, h\right)|f((\epsilon+i t) \lambda / n)-\widetilde{f}(i t \lambda / n)|^{2} \\
& =\int_{[0, \infty)} d\left(E_{A}(\lambda) h, h\right) \int_{-T}^{T} d t|f((\epsilon+i t) \lambda / n)-\tilde{f}(i t \lambda / n)|^{2}
\end{array}
$$

for each $n \in \mathbb{N}$ and $h \in \mathfrak{H}$. For any $\lambda \in[0, \infty)$ we have

$$
\lim _{\epsilon \rightarrow+0} \int_{-T}^{T} d t|f((\epsilon+i t) \lambda / n)-\widetilde{f}(i t \lambda / n)|^{2}=0
$$

which yields

$$
\lim _{\epsilon \rightarrow+0} \int_{-T}^{T} d t\|f((\epsilon+i t) A / n) h-\tilde{f}(i t A / n) h\|^{2}=0
$$

for each $n \in \mathbb{N}$ and $h \in \mathfrak{H}$. Since also

$$
\lim _{\epsilon \rightarrow+0} \int_{-T}^{T} d t\|g((\epsilon+i t) B / n) h-\widetilde{g}(i t B / n) h\|^{2}=0
$$

holds for each $n \in \mathbb{N}$ and $h \in \mathfrak{H}$ we immediately find that

$\lim _{\epsilon \rightarrow+0} \int_{-T}^{T} d t\left\|(f((\epsilon+i t) A / n) g((\epsilon+i t) B / n))^{n} h-(\widetilde{f}(i t A / n) \widetilde{g}(i t B / n))^{n} h\right\|^{2}=0$

for each $n \in \mathbb{N}$ and $h \in \mathfrak{H}$. Hence

$$
\begin{gathered}
0=\lim _{\epsilon \rightarrow+0} \int_{-T}^{T} d t\left\|F_{n}(\epsilon+i t) h-(\widetilde{f}(i t A / n) \widetilde{g}(i t B / n))^{n} h\right\|^{2} \\
=\int_{-T}^{T} d t\left\|F_{n}(i t) h-(\widetilde{f}(i t A / n) \widetilde{g}(i t B / n))^{n} h\right\|^{2}
\end{gathered}
$$

for each $n \in \mathbb{N}$ and $h \in \mathfrak{H}$ which yields $F_{n}(i t)=(\widetilde{f}(i t A / n) \widetilde{g}(i t B / n))^{n}$ for a.e. $t \in \mathbb{R}$ and $n \in \mathbb{N}$.

Using the standard Kato function $f_{k}(x)$, see above, we get

$$
\lim _{n \rightarrow+\infty} \int_{-T}^{T}\left\|\left((I+i t A / k n)^{-k}(I+i t B / k n)^{-k}\right)^{n} h-e^{-i t C} h\right\| d t=0
$$

for any $h \in \mathfrak{H}$ and $T>0$. We note that for the particular case $k=1$ Lapidus demonstrated in [15] that (1.10) holds uniformly in $t \in[0, T]$ for any $T>0$. By Theorem 3.3 one gets that formula (1.10) is valid in a weaker topology than in [15]. This discrepancy will be clarified in the next section. 
The set of holomorphic Kato functions was characterized in [7]. For the sake of completeness we recall these results here:

Theorem 3.4 ([7, Theorem 5.1]). If $f$ is a holomorphic Kato function, then

(i) there is an at most countable set of complex numbers $\left\{\xi_{k}\right\}_{k}, \xi_{k} \in \mathbb{C}_{\text {right }}$ with $\Im \mathrm{m}\left(\xi_{k}\right) \geq 0$ satisfying the condition

$$
\varkappa:=4 \sum_{k} \frac{\Re \mathrm{e}\left(\xi_{k}\right)}{\left|\xi_{k}\right|^{2}} \leq 1
$$

(ii) there is a Borel measure $\nu$ defined on $\overline{\mathbb{R}}_{+}=[0, \infty)$ obeying $\nu(\{0\})=0$ and

$$
\int_{\mathbb{R}_{+}} \frac{1}{1+t^{2}} d \nu(t)<\infty
$$

such that the limit $\beta:=\lim _{x \rightarrow+0} \frac{2}{\pi} \int_{\mathbb{R}_{+}} \frac{1}{x^{2}+t^{2}} d \nu(t)$ exists and satisfies the condition $\beta \leq 1-\varkappa$;

(iii) the Kato function $f$ admits the representation

$$
f(x)=D(x) \exp \left\{-\frac{2 x}{\pi} \int_{\mathbb{R}_{+}} \frac{1}{x^{2}+t^{2}} d \nu(t)\right\} e^{-\alpha x}, \quad x \in \mathbb{R}_{+},
$$

where $\alpha:=1-\varkappa-\beta$ and $D(x)$ is a Blaschke-type product given by

$$
D(x):=\prod_{k} \frac{x^{2}-2 x \Re \mathrm{e}\left(\xi_{k}\right)+\left|\xi_{k}\right|^{2}}{x^{2}+2 x \Re \mathrm{e}\left(\xi_{k}\right)+\left|\xi_{k}\right|^{2}}, \quad x \in \mathbb{R}_{+} .
$$

The factor $D(x)$ is absent if the set $\left\{\xi_{k}\right\}_{k}$ is empty; in that case we set $\varkappa:=0$.

Conversely, if a real function $f$ admits the representation (3.2) such that the assumptions (i) and (ii) are satisfied and the condition $\alpha+\varkappa+\beta=1$ holds, then $f$ is a holomorphic Kato function and its holomorphic extension to $\mathbb{C}_{\text {right }}$ is given by

$$
f(z)=D(z) \exp \left\{-\frac{2 z}{\pi} \int_{\mathbb{R}_{+}} \frac{1}{z^{2}+t^{2}} d \nu(t)\right\} e^{-\alpha z}, \quad z \in \mathbb{C}_{\text {right }} .
$$

Above we have indicated several standard holomorphic Kato functions such as $f(z)=e^{-z}, f_{k}(z)=(1+i z / k)^{-k}, k \in \mathbb{N}, z \in \mathbb{C}_{\text {right }}$. The last theorem allows us to give examples of some non-standard holomorphic Kato functions.

1. If a holomorphic Kato function $f(\cdot)$ has no zeros in $\mathbb{C}_{\text {right }}$ and $\nu \equiv 0$, then $f(z)=e^{-z}, z \in \mathbb{C}_{\text {right }}$, where $\alpha=1$ follows from condition $\alpha=1-\varkappa-\beta$ where $\varkappa=\beta=0$. Obviously, we have $\widetilde{f}(i y)=\lim _{\epsilon \rightarrow+0} f(\epsilon+i y)=e^{-i y}$ for $y \in \mathbb{R}$.

2. If a holomorphic Kato function $f(\cdot)$ has zeros and the measure $\nu \equiv 0$, then $f(\cdot)$ is of the form $f(z)=D(z) e^{-\alpha z}$, where the Blaschke-type product $D(z)$ is given by (3.3). In particular, if $n=1$ we find the representation

$$
f(z)=\frac{z^{2}-2 z \Re \mathrm{e}(\xi)+|\xi|^{2}}{z^{2}+2 z \Re \mathrm{e}(\xi)+|\xi|^{2}} e^{-\alpha z}, \quad z \in \mathbb{C}_{\text {right }},
$$


where $\xi \in \mathbb{C}_{\text {right }}$ is such that

$$
\alpha+4 \frac{\Re \mathrm{e}(\xi)}{|\xi|^{2}}=1 .
$$

This gives the representation

$$
f(z)=\frac{z^{2}-2 \eta\left(z-\frac{2}{1-\alpha}\right)}{z^{2}+2 \eta\left(z+\frac{2}{1-\alpha}\right)} e^{-\alpha z}, \quad z \in \mathbb{C}_{\text {right }},
$$

$0<\eta \leq \frac{4}{1-\alpha}, 0 \leq \alpha \leq 1$, where we have denoted $\xi=\eta+i \tau, \eta>0$, and $\tau=\sqrt{\frac{4}{(1-\alpha)^{2}}-\left(\eta-\frac{2}{1-\alpha}\right)^{2}}$. We have

$$
\widetilde{f}(i y)=\lim _{\epsilon \rightarrow+0} f(\epsilon+i y)=\frac{y^{2}+4 \eta \frac{1}{1-\alpha}+2 i \eta y}{y^{2}-4 \eta \frac{1}{1-\alpha}+2 i \eta y} e^{-i \alpha y}, \quad y \in \mathbb{R} .
$$

3. If a holomorphic Kato function $f(z)$ has no zeros and the measure $\nu$ is atomic, then $f(z)$ admits the representation

$$
f(z)=\exp \left\{-\frac{2 z}{\pi} \sum_{l} \frac{1}{z^{2}+s_{l}^{2}} \nu\left(\left\{s_{l}\right\}\right)\right\} e^{-\alpha z}, \quad z \in \mathbb{C}_{\text {right }},
$$

where $\left\{s_{l}\right\}_{l}$ is the point where $\nu\left(\left\{s_{l}\right\}\right) \neq 0$. In the particular case when $d \nu(t)=c \delta(t-s) d t, s>0$, we have

$$
f(z)=\exp \left\{-\frac{2 z c}{\pi} \frac{1}{z^{2}+s^{2}}\right\} e^{-\alpha z},
$$

and $\alpha+\frac{2 c}{\pi} \frac{1}{s^{2}}=1$, which yields $c=\frac{1}{2}(1-\alpha) \pi s^{2}$ and

$$
f(z):=\exp \left\{-z(1-\alpha) \frac{s^{2}}{z^{2}+s^{2}}\right\} e^{-\alpha z}, \quad z \in \mathbb{C}_{\text {right }} .
$$

One gets

$$
\widetilde{f}(i y)= \begin{cases}\lim _{\epsilon \rightarrow+0} f(\epsilon+i y)=\exp \left\{i y(1-\alpha) \frac{s^{2}}{y^{2}-s^{2}}\right\} e^{-i \alpha y} & y \neq \pm s \\ 0 & y= \pm s\end{cases}
$$

where $y \in \mathbb{R}$.

4. If a holomorphic Kato function $f(z)$ has no zeros and the measure $\nu$ is absolutely continuous, that is, $d \nu(t)=h(t) d t, h(t)\left(1+t^{2}\right)^{-1} \in L^{1}\left(\mathbb{R}_{+}\right)$, then $f(z)$ admits the representation

$$
f(z)=\exp \left\{-\frac{2 z}{\pi} \int_{0}^{\infty} \frac{h(t)}{z^{2}+t^{2}} d t\right\} e^{-\alpha z}, \quad z \in \mathbb{C}_{\text {right }},
$$

such that

$$
\alpha+\lim _{x \rightarrow+0} \frac{2}{\pi} \int_{0}^{\infty} \frac{h(t)}{x^{2}+t^{2}} d t=1
$$


If $h(\cdot)$ is Hölder continuous, then $\lim _{\epsilon \rightarrow+0} \frac{2(\epsilon+i y)}{\pi} \int_{0}^{\infty} \frac{h(t)}{(\epsilon+i y)^{2}+t^{2}} d t$ exists for each $y \in \mathbb{R}$ and one gets

$$
\widetilde{f}(i y)=\exp \left\{-\lim _{\epsilon \rightarrow+0} \frac{2(\epsilon+i y)}{\pi} \int_{0}^{\infty} \frac{h(t)}{(\epsilon+i y)^{2}+t^{2}}\right\} e^{-i y}, \quad y \in \mathbb{R} .
$$

In particular, if $f(x)=\left(1+\frac{x}{k}\right)^{-k}, x \in \mathbb{R}_{+}, k \in \mathbb{N}$, then

$$
f(z)=\exp \left\{-\frac{k z}{\pi} \int_{\mathbb{R}_{+}} \frac{1}{z^{2}+t^{2}} \ln \left(1+\frac{t^{2}}{k^{2}}\right) d t\right\}
$$

for $z \in \mathbb{C}_{\text {right }}$ and $\tilde{f}(i y)=(1+i y / k)^{-k}, y \in \mathbb{R}, k \in \mathbb{N}$.

\section{Ichinose's result revisited}

Recall that the notion of admissible functions was introduced in [6, Definition 1].

Definition 4.1. A Borel measurable function $\phi: \mathbb{R}_{+} \longrightarrow \mathbb{C}$ is called admissible if the conditions

$$
|\phi(y)| \leq 1, \quad y \in[0, \infty), \quad \phi(0)=1, \quad \phi^{\prime}(0)=-i
$$

are satisfied.

We set $\phi_{\mathcal{R}}(y):=\Re \mathrm{e}(\phi(y))$ and $\phi_{\mathcal{I}}(y):=\Im \mathrm{m}(\phi(y)), y \in \mathbb{R}_{+}$. Obviously we have

$$
\left|\phi_{\mathcal{R}}(y)\right| \leq 1, \quad y \in \mathbb{R}_{+}, \quad \phi_{\mathcal{R}}(0)=1 \quad \text { and } \quad \phi_{\mathcal{R}}^{\prime}(0)=0
$$

as well as

$$
\left|\phi_{\mathcal{I}}(y)\right| \leq 1, \quad y \in \mathbb{R}_{+}, \quad \phi_{\mathcal{I}}(0)=0 \quad \text { and } \quad \phi_{\mathcal{I}}^{\prime}(0)=-1 .
$$

Let $\Sigma:=\left\{y \in \mathbb{R}_{+}: \phi(y)=0\right\}$ and $\Omega:=\mathbb{R} \backslash \Sigma$. We set

$$
\varphi(y):= \begin{cases}\frac{1}{\phi(y)}, & y \in \Omega \\ 1, & y \in \Sigma .\end{cases}
$$

Notice that $\chi_{\Omega}(y)=\varphi(y) \phi(y), y \in \Omega$, where $\chi_{\Omega}(\cdot)$ is the characteristic function of $\Omega$. The function $\varphi$ obeys

$$
|\varphi(y)| \geq 1, \quad y \in \mathbb{R}_{+}, \quad \varphi(0)=1, \quad \varphi^{\prime}(0)=i .
$$

Moreover, we find

and

$$
\varphi_{\mathcal{R}}(y):=\Re \mathrm{R}(\varphi(y))= \begin{cases}\frac{\phi_{\mathcal{R}}(y)}{|\phi(y)|^{2}}, & y \in \Omega \\ 1, & y \in \Sigma\end{cases}
$$

as well as

$$
\varphi_{\mathcal{I}}(y):=\Im \mathrm{m}(\varphi(y))= \begin{cases}-\frac{\phi_{\mathcal{I}}(y)}{|\phi(y)|^{2}}, & y \in \Omega \\ 0, & y \in \Sigma\end{cases}
$$

$$
\varphi_{\mathcal{R}}(0)=1, \quad \varphi_{\mathcal{R}}^{\prime}(0)=0 \quad \text { and } \quad \varphi_{\mathcal{I}}(0)=0, \quad \varphi_{\mathcal{I}}^{\prime}(0)=1
$$


Let $E_{\tau}:=\chi_{\Omega}(\tau A), \tau>0$. Obviously, $E_{\tau}$ is an orthogonal projection. We consider the operator-valued function

$$
K(\tau):=\frac{\varphi(\tau A)-I}{\tau}+E_{\tau} \frac{I-\psi(\tau B)}{\tau} E_{\tau}, \quad \tau>0
$$

We note that

$$
K_{\mathcal{R}}(\tau):=\Re \mathrm{e}(K(\tau))=\frac{\varphi_{\mathcal{R}}(\tau A)-I}{\tau}+E_{\tau} \frac{I-\psi_{\mathcal{R}}(\tau B)}{\tau} E_{\tau}
$$

and

$$
K_{\mathcal{I}}(\tau):=\Im \mathrm{m}(K(\tau))=\frac{\varphi_{\mathcal{I}}(\tau A)-E_{\tau} \psi_{\mathcal{I}}(\tau B) E_{\tau}}{\tau} .
$$

If $\phi_{\mathcal{I}}(y) \leq 0$ and $\psi_{\mathcal{I}}(y) \leq 0, y \in \mathbb{R}_{+}$, then $K_{\mathcal{I}}(\tau) \geq 0$. Furthermore, we set

$$
L_{\gamma}(\tau):=\gamma K_{\mathcal{R}}(\tau)+K_{\mathcal{I}}(\tau), \quad \gamma \in[0,1] .
$$

Let us introduce the functions

$$
f_{\gamma}(y):=\gamma\left(\varphi_{\mathcal{R}}(y)-1\right)+\varphi_{\mathcal{I}}(y), \quad y \in \mathbb{R}_{+},
$$

and

$$
g_{\gamma}(y):=\gamma\left(1-\psi_{\mathcal{R}}(y)\right)-\psi_{\mathcal{I}}(y), \quad y \in \mathbb{R}_{+},
$$

for $\gamma \in[0,1]$. If $\phi(\cdot)$ and $\psi(\cdot)$ are admissible functions, then

$$
f_{\gamma}(0)=0, \quad f_{\gamma}^{\prime}(0)=1 \quad \text { and } \quad g_{\gamma}(0)=0, \quad g_{\gamma}^{\prime}(0)=1 .
$$

Using the functions $f_{\gamma}(\cdot)$ and $g_{\gamma}(\cdot)$ one gets the representation

$$
L_{\gamma}(\tau)=\frac{f_{\gamma}(\tau A)}{\tau}+E_{\tau} \frac{g_{\gamma}(\tau B)}{\tau} E_{\tau}
$$

If $\phi_{\mathcal{R}}(y) \geq 0$ and $\phi_{\mathcal{I}}(y) \leq 0$ for $y \in \mathbb{R}_{+}$, then

$$
f_{\gamma}(y)= \begin{cases}\frac{\gamma \phi_{\mathcal{R}}(y)\left(I-\phi_{\mathcal{R}}(y)\right)-\phi_{\mathcal{I}}(y)\left(1+\gamma \phi_{\mathcal{I}}(y)\right)}{|\phi(y)|^{2}}, & y \in \Omega \\ 0, & y \in \Sigma\end{cases}
$$

yields $f_{\gamma}(y) \geq 0$ for $y \in \mathbb{R}_{+}$and $\gamma \in[0,1]$. Hence $f_{\gamma}(\tau A) \geq 0$ for $\tau>0$ and $\gamma \in[0,1]$. Similarly, if $\psi_{\mathcal{I}}(y) \leq 0$ for $y \in \mathbb{R}_{+}$, then $g_{\gamma}(y) \geq 0$ for $y \in \mathbb{R}_{+}$which implies $g_{\gamma}(\tau B) \geq 0$ for $\tau>0$ and $\gamma \in[0,1]$. Hence one has $L_{\gamma}(\tau) \geq 0$ and $\tau>0$ and $\gamma \in[0,1]$, which shows that $\left(\mu I+L_{\gamma}(\tau)\right)^{-1}$ exists and is bounded for $\mu>0$, $\tau>0$ and $\gamma \in[0,1]$.

Lemma 4.2. Let $A$ and $B$ be non-negative self-adjoint operators such that the intersection $\operatorname{dom}\left(A^{1 / 2}\right) \cap \operatorname{dom}\left(B^{1 / 2}\right)$ is dense in $\mathfrak{H}$. If $f(\cdot): \mathbb{R}_{+} \longrightarrow \mathbb{R}$ and $g(\cdot)$ : $\mathbb{R}_{+} \longrightarrow \mathbb{R}$ are finite-valued non-negative Borel measurable functions satisfying

$$
f(0)=0, \quad f^{\prime}(0)=1 \quad \text { and } \quad g(0)=0, \quad g^{\prime}(0)=1,
$$

and

$$
0 \leq g(y) \leq 1, \quad y \in \mathbb{R}_{+},
$$

then

$$
\text { S- } \lim _{\tau \rightarrow+0}(\mu I+L(\tau))^{-1}=(\mu I+C)^{-1}
$$


for $\mu>0$ where

$$
L(\tau):=\frac{1}{\tau} f(\tau A)+\frac{1}{\tau} E_{\tau} g(\tau B) E_{\tau}, \quad \tau>0 .
$$

and $E_{\tau}:=\chi_{\Omega}(\tau A), \Omega \supseteq \operatorname{supp}(f) \cup\{0\}, \operatorname{supp}(f):=\left\{y \in \mathbb{R}_{+}: f(y)>0\right\}$.

Proof. Since $f(\cdot)$ takes only finite values the operator $f(\tau A), \tau>0$, is densely defined. Moreover, the operator $g(\tau B)$ is bounded. Hence the operator $L(\tau)$ is well-defined. We set

$$
p(y):=\frac{1}{1+f(y)} \quad \text { and } \quad q(y):=1-g(y), \quad y \in \mathbb{R}_{+} .
$$

We note that

$$
0 \leq p(y) \leq 1, \quad y \in \mathbb{R}_{+}, \quad p(0)=1, \quad \text { and } \quad p^{\prime}(0)=-1
$$

as well as

$$
0 \leq q(y) \leq 1, \quad y \in \mathbb{R}_{+}, \quad q(0)=1, \quad \text { and } \quad q^{\prime}(0)=-1
$$

Hence $p(\cdot)$ and $q(\cdot)$ are Kato functions. We have

$$
L(\tau)=p(\tau A)^{-1 / 2}\left(\frac{I-p(\tau A)}{\tau}+\sqrt{p(\tau A)} E_{\tau} \frac{I-q(\tau B)}{\tau} E_{\tau} \sqrt{p(\tau A)}\right) p(\tau A)^{-1 / 2} .
$$

Let

$$
\widehat{F}(\tau):=p(\tau A)^{1 / 2} E_{\tau} q(\tau B) E_{\tau} p(\tau A)^{1 / 2}, \quad \tau \geq 0 .
$$

Since $p(y)=1$ for $y \in \Sigma:=\mathbb{R} \backslash \Omega \subseteq \operatorname{ker}(f), \operatorname{ker}(f):=\left\{y \in \mathbb{R}_{+}: f(y)=0\right\}$, we find

$$
L(\tau)=p(\tau A)^{-1 / 2} E_{\tau} \widehat{S}_{\tau} E_{\tau} p(\tau A)^{-1 / 2},
$$

where

$$
\widehat{S}_{\tau}:=\frac{I-\widehat{F}(\tau)}{\tau}, \quad \tau>0
$$

Hence the representation

$$
(\mu I+L(\tau))^{-1}=\sqrt{p(\tau A)}\left(\mu p(\tau A)+E_{\tau} \widehat{S}_{\tau} E_{\tau}\right)^{-1} \sqrt{p(\tau A)}
$$

holds. Since $0 \leq p(\tau A) \leq I$ we obtain

$$
\sqrt{p(\tau A)}\left(\mu I+E_{\tau} \widehat{S}_{\tau} E_{\tau}\right)^{-1} \sqrt{p(\tau A)} \leq(\mu I+L(\tau))^{-1}
$$

for $\mu>0$. By the formula

$$
\left.\left(\mu I+E_{\tau} \widehat{S}_{\tau} E_{\tau}\right)\right)^{-1}=\frac{1}{\mu} E_{\tau}^{\perp}+E_{\tau}\left(\mu E_{\tau}+E_{\tau} \widehat{S}_{\tau} E_{\tau}\right)^{-1} E_{\tau}
$$

we get

$$
\sqrt{p(\tau A)} E_{\tau}\left(\mu E_{\tau}+E_{\tau} \widehat{S}_{\tau} E_{\tau}\right)^{-1} E_{\tau} \sqrt{p(\tau A)} \leq(\mu I+L(\tau))^{-1}
$$


for $\tau>0$. Setting $\widehat{p}(y):=p(y) \chi_{\Omega}(y), y \in \mathbb{R}_{+}$, we find the representation $\widehat{F}(\tau)=$ $\sqrt{\widehat{p}(\tau A)} q(\tau B) \sqrt{\widehat{p}(\tau A)}, \tau>0$. Since $\widehat{p}(\cdot)$ and $q(\cdot)$ are Kato functions we obtain

$$
\text { s- } \lim _{\tau \rightarrow+0}\left(\mu I+\widehat{S}_{\tau}(\tau)\right)^{-1}=(\mu I+C)^{-1}
$$

for $\mu>0$ using [3] and [14]. Taking into account formula (4.13) we find

$$
\text { s- } \lim _{\tau \rightarrow+0} E_{\tau}\left(\mu E_{\tau}+E_{\tau} \widehat{S}_{\tau} E_{\tau}\right)^{-1} E_{\tau}=(\mu I+C)^{-1}
$$

for $\mu>0$. From (4.12) and (4.14) we finally get

$$
\left((\mu I+C)^{-1} h, h\right) \leq \liminf _{\tau \rightarrow+0}\left((\mu I+L(\tau))^{-1} h, h\right)^{-1}
$$

for $h \in \mathfrak{H}, \mu>0$. Moreover, from (4.5) we find

$$
L(\tau) \geq \frac{f(\tau A)}{\tau} E_{A}([0, a))+E_{\tau} \frac{g(\tau B)}{\tau} E_{B}([0, b)) E_{\tau}, \quad a, b \in(0, \infty),
$$

which gives the estimate

$$
(\mu I+L(\tau))^{-1} \leq\left(\mu I+\frac{f(\tau A)}{\tau} E_{A}([0, a))+E_{\tau} \frac{g(\tau B)}{\tau} E_{B}([0, b)) E_{\tau}\right)^{-1}
$$

for $\mu>0$ and $a, b \in(0, \infty)$. Using $\mathrm{s}^{-\lim _{\tau \rightarrow+0}} E_{\tau}=I$ we obtain

$$
\limsup _{\tau \rightarrow+0}\left((\mu I+L(\tau))^{-1} h, h\right) \leq\left(\left(\mu I+A E_{A}([0, a))+B E_{B}([0, b))\right)^{-1} h, h\right) .
$$

for $h \in \mathfrak{H}, \mu>0$ and $a, b \in(0, \infty)$. Since $a, b \in(0, \infty)$ are arbitrary we obtain

$$
\limsup _{\tau \rightarrow+0}\left((\mu I+L(\tau))^{-1} h, h\right) \leq\left((\mu I+C)^{-1} h, h\right)
$$

for $h \in \mathfrak{H}, \mu>0$. Hence

$$
\mathrm{w}-\lim _{\tau \rightarrow+0}(\mu I+L(\tau))^{-1}=(\mu I+C)^{-1}
$$

for $\mu>0$, and consequently,

$$
\text { W- } \lim _{\tau \rightarrow+0}(\mu I+L(\tau))^{-1 / 2}=(\mu I+C)^{-1 / 2}
$$

for $\mu>0$. From (4.15) and (4.16) we immediately get (4.18).

Lemma 4.3. Let $A$ and $B$ be non-negative self-adjoint operators such that the intersection $\operatorname{dom}\left(A^{1 / 2}\right) \cap \operatorname{dom}\left(B^{1 / 2}\right)$ is dense in $\mathfrak{H}$. If $\phi$ and $\psi$ are admissible functions such that

$$
\phi_{\mathcal{R}}(y) \geq 0, \quad \phi_{\mathcal{I}}(y) \leq 0 \quad \text { and } \quad \psi_{\mathcal{I}}(y) \leq 0, \quad y \in \mathbb{R}_{+},
$$

then the self-adjoint operators $L_{\gamma}(\tau), \tau>0$ are well-defined and non-negative, and it holds

$$
\text { S- } \lim _{\tau \rightarrow+0}\left(\mu I+L_{\gamma}(\tau)\right)^{-1}=(\mu I+C)^{-1}
$$

for $\mu>0$ and $\gamma \in[0,1]$. 
Proof. One easily verifies that the functions $f_{\gamma}(\cdot)$ and $g_{\gamma}(\cdot)$ defined by $(4.2)$ and (4.3) satisfy the assumptions (4.6) and (4.7) for each $\gamma \in[0,1]$. Setting $\Omega:=$ $\operatorname{supp}(\phi):=\left\{y \in \mathbb{R}_{+}: \phi(y) \neq 0\right\}$ we find $\Omega \supseteq \operatorname{supp}\left(f_{\gamma}\right) \cup\{0\}$ for $\gamma=[0,1]$. Moreover, the definition of $L_{\gamma}(\tau)$ given by (4.5) coincides with that one of $L(\tau)$ for each $\tau>0$ and $\gamma \in[0,1]$, see (4.9). Applying Lemma 4.2 we arrive at the sought conclusion.

For purposes of the next statement we introduce the operators

$$
M_{\gamma}(\tau):=L_{\gamma}(\tau)+(1+\gamma) \varphi_{\mathcal{R}}(\tau A)+(1-\gamma) \varphi_{\mathcal{I}}(\tau A)
$$

with $\tau>0$ and $\gamma \in[0,1]$. Since $L_{\gamma}(\tau) \geq 0, \varphi_{\mathcal{R}}(\tau A) \geq 0$ and $\varphi_{\mathcal{I}}(\tau A) \geq 0$ we get $M_{\gamma}(\tau) \geq 0$ for $\gamma \in[0,1]$.

Lemma 4.4. Let $A$ and $B$ be non-negative self-adjoint operators such that the intersection $\operatorname{dom}\left(A^{1 / 2}\right) \cap \operatorname{dom}\left(B^{1 / 2}\right)$ is dense in $\mathfrak{H}$. If $\phi$ and $\psi$ are admissible functions such that the conditions (4.17) are satisfied, then $M_{\gamma}(\tau) \geq 0$ and

$$
\text { S- } \lim _{\tau \rightarrow+0}\left(\mu I+M_{\gamma}(\tau)\right)^{-1}=((1+\mu+\gamma) I+C)^{-1}
$$

holds for $\mu>0$ and $\gamma \in[0,1]$.

Proof. We note that $M_{\gamma}(\tau) \geq L_{\gamma}(\tau)+(1+\gamma) \varphi_{\mathcal{R}}(\tau A) \geq 0$ for $\tau>0$ and $\gamma \in[0,1]$. Let $\Omega_{\mathcal{R}}^{(n)}:=\left\{y \in \mathbb{R}: \varphi_{\mathcal{R}}(y) \leq n\right\}$. We set

$$
\varphi_{\mathcal{R}}^{(n)}(y):= \begin{cases}\varphi_{\mathcal{R}}(y), & y \in \Omega_{\mathcal{R}}^{(n)} \\ n, & y \in \mathbb{R}_{+} \backslash \Omega_{\mathcal{R}}^{(n)}\end{cases}
$$

for any $n \in \mathbb{N}$. Obviously we have $0 \leq \varphi_{\mathcal{R}}^{(n)}(y) \leq n, y \in \mathbb{R}_{+}$, and $0 \leq \varphi_{\mathcal{R}}^{(n)}(y) \leq$ $\varphi_{\mathcal{R}}(y), y \in \mathbb{R}_{+}$. Therefore one obtains

$$
M_{\gamma}(\tau) \geq L_{\gamma}(\tau)+(1+\gamma) \varphi_{\mathcal{R}}^{(n)}(\tau A) \geq 0
$$

for $\tau>0$ and $\gamma \in[0,1]$ which yields

$$
\left(\mu I+M_{\gamma}(\tau)\right)^{-1} \leq\left(\mu I+L_{\gamma}(\tau)+(1+\gamma) \varphi_{\mathcal{R}}^{(n)}(\tau A)\right)^{-1},
$$

for $\mu>0$ and $\gamma \in[0,1]$. Since $\operatorname{s}^{-\lim _{\tau \rightarrow+0}} \varphi_{\mathcal{R}}^{(n)}(\tau A)=I$ we obtain from Lemma 4.3 that

$$
\text { S- } \lim _{\tau \rightarrow+0}\left(\mu I+L_{\gamma}(\tau)+(1+\gamma) \varphi_{\mathcal{R}}^{(n)}(\tau A)\right)^{-1}=((1+\mu+\gamma) I+C)^{-1} .
$$

Hence

$$
\limsup _{\tau \rightarrow+0}\left(\left(\mu I+M_{\gamma}(\tau)\right)^{-1} h, h\right) \leq\left(((1+\mu+\gamma) I+C)^{-1} h, h\right)
$$

for $\mu>0$ and $\gamma \in[0,1]$. Furthermore, we note that

$$
M_{\gamma}(\tau) \leq(1+\gamma) I+L_{\gamma}(\tau)+(1+\gamma) \rho(\tau A)
$$


where $\rho(y):=\varphi_{\mathcal{R}}(y)+\varphi_{\mathcal{I}}(y)-1, y \in \mathbb{R}_{+}$. One has $\rho(0)=0$ and $\rho^{\prime}(0)=1$. Hence we find

$$
M_{\gamma}(\tau) \leq(1+\gamma) I+L_{\gamma}(\tau)+\tau_{0}(1+\gamma) \frac{\rho(\tau A)}{\tau}
$$

for $0<\tau \leq \tau_{0}$. By

$$
1+\rho(y)=\frac{\phi_{\mathcal{R}}(y)-\phi_{\mathcal{I}}(y)}{|\phi(y)|^{2}} \geq \frac{\phi_{\mathcal{R}}^{2}(y)+\phi_{\mathcal{I}}^{2}(y)}{|\phi(y)|^{2}} \geq 1, \quad y \in \mathbb{R}_{+},
$$

we find $\rho(y) \geq 0, y \in \mathbb{R}_{+}$. We set

$$
\widetilde{f}_{\gamma}(y):=\frac{1}{\left(1+\tau_{0}+\tau_{0} \gamma\right)}\left(f_{\gamma}(y)+\tau_{0}(1+\gamma) \rho(y)\right), \quad y \in \mathbb{R}_{+}, \quad \tau_{0}>0,
$$

where $f_{\gamma}(y)$ is given by $(4.2)$. It holds $\widetilde{f}_{\gamma}(0)=0$ and $\widetilde{f}_{\gamma}^{\prime}(0)=1$ as well as $\widetilde{f}_{\gamma}(y)=0$ for $y \in \Sigma$. One gets

$$
L_{\gamma}(\tau)+\tau_{0}(1+\gamma) \frac{\rho(\tau A)}{\tau} \leq\left(1+\tau_{0}+\gamma \tau_{0}\right)\left(\frac{\tilde{f}_{\gamma}(\tau A)}{\tau}+E_{\tau} \frac{g_{\gamma}(\tau B)}{\tau} E_{\tau}\right)
$$

for $\gamma \in[0,1]$ and $\tau \in\left(0, \tau_{0}\right]$ where $g_{\gamma}(y)$ is given by (4.3). Setting

we obtain

$$
\widetilde{L}_{\gamma}(\tau):=\frac{\tilde{f}_{\gamma}(\tau A)}{\tau}+E_{\tau} \frac{g_{\gamma}(\tau B)}{\tau} E_{\tau}, \quad \tau>0, \quad \gamma \in[0,1],
$$

$$
M_{\gamma}(\tau) \leq(1+\gamma) I+\left(1+\tau_{0}+\gamma \tau_{0}\right) \widetilde{L}_{\gamma}(\tau), \quad \tau>0, \quad \gamma \in[0,1],
$$

which yields

$\left((1+\mu+\gamma) I+\left(1+\tau_{0}+\gamma \tau_{0}\right) \widetilde{L}_{\gamma}(\tau)\right)^{-1} \leq\left(\mu I+M_{\gamma}(\tau)\right)^{-1}, \quad \mu>0, \quad 0<\tau \leq \tau_{0}$, and $\gamma \in[0,1]$. Let $\lambda:=\frac{1+\mu+\gamma}{1+\tau_{0}+\gamma \tau_{0}}$, we find

$$
\left(\lambda I+\widetilde{L}_{\gamma}(\tau)\right)^{-1} \leq\left(1+\tau_{0}+\gamma \tau_{0}\right)\left(\mu I+M_{\gamma}(\tau)\right)^{-1}
$$

for $\mu>0,0<\tau \leq \tau_{0}$ and $\gamma \in[0,1]$. Applying Lemma 4.2 we immediately get that

$$
\left((\lambda I+C)^{-1} h, h\right) \leq\left(1+\tau_{0}+\gamma \tau_{0}\right) \liminf _{\tau \rightarrow+0}\left(\left(\mu+M_{\gamma}(\tau)\right)^{-1} h, h\right)
$$

for $\mu>0, \tau_{0}>0, \gamma \in[0,1]$ and $h \in \mathfrak{H}$. Since $\tau_{0}>0$ is arbitrary we finally obtain

$$
\left(((1+\mu+\gamma) I+C)^{-1} h, h\right) \leq \liminf _{\tau \rightarrow+0}\left(\left(\mu I+M_{\gamma}(\tau)\right)^{-1} h, h\right)
$$

for $\mu>0, \gamma \in[0,1]$ and $h \in \mathfrak{H}$. From (4.21) and (4.22) we deduce that

$$
\mathrm{w}-\lim _{\tau \rightarrow+0}\left(\mu I+M_{\alpha}(\tau)\right)^{-1}=((1+\mu+\gamma) I+C)^{-1}
$$

holds for $\mu>0$ and $\gamma \in[0,1]$. Since the relation (4.23) is valid for every $\mu>0$ we get

$$
\text { w- } \lim _{\tau \rightarrow+0}\left(\mu I+M_{\alpha}(\tau)\right)^{-1 / 2}=((1+\mu+\gamma) I+C)^{-1 / 2}
$$

for $\mu>0$ which yields

$$
\mathrm{s}-\lim _{\tau \rightarrow+0}\left(\mu I+M_{\alpha}(\tau)\right)^{-1 / 2}=((1+\mu+\gamma) I+C)^{-1 / 2}
$$


for $\mu>0$. The last relation proves (4.20).

Let us introduce the operator-valued function

$$
T(\tau):=\frac{1}{\sqrt{I+M_{0}(\tau)}}\left(K_{\mathcal{R}}(\tau)+\varphi_{\mathcal{R}}(\tau A)-\varphi_{\mathcal{I}}(\tau A)\right) \frac{1}{\sqrt{I+M_{0}(\tau)}}, \quad \tau>0,
$$

where $M_{0}(\tau)=K_{\mathcal{I}}(\tau)+\varphi_{\mathcal{R}}(\tau A)+\varphi_{\mathcal{I}}(\tau A) \geq 0$, see (4.1), (4.5) and (4.19).

Lemma 4.5. Let $A$ and $B$ be non-negative self-adjoint operators in a separable Hilbert space $\mathfrak{H}$ such that $\operatorname{dom}\left(A^{1 / 2}\right) \cap \operatorname{dom}\left(B^{1 / 2}\right)$ is dense in $\mathfrak{H}$. If $\phi$ and $\psi$ are admissible functions such that the conditions (4.17) are satisfied, then $T(\tau) \geq-I$, $\tau>0$, and

$$
\text { S- } \lim _{\tau \rightarrow+0}(i I+T(\tau))^{-1}=\left(i I+(2 I+C)^{-1}\right)^{-1},
$$

where $T(\tau)$ is defined by (4.24).

Proof. Since $M_{\gamma}(\tau) \geq 0$ for $\gamma \in[0,1]$ and $\tau>0$ we find $I+T(\tau) \geq 0$, which yields $T(\tau) \geq-I$. Hence $\gamma T(\tau) \geq-\gamma I$ holds for $\gamma \in[0,1]$, and therefore the operator $I+\gamma T(\tau)$ is boundedly invertible for $\gamma \in[0,1)$ and we have the representation

$$
\left(I+M_{\gamma}(\tau)\right)^{-1}=\frac{1}{\sqrt{I+M_{0}(\tau)}}(I+\gamma T(\tau))^{-1} \frac{1}{\sqrt{I+M_{0}(\tau)}}
$$

for $\gamma \in[0,1)$. Setting $\gamma=0$ we find from Lemma 4.4 that

$$
\text { S- } \lim _{\tau \rightarrow+0} \frac{1}{\sqrt{I+M_{0}(\tau)}}=(2 I+C)^{-1 / 2} \text {. }
$$

Since $\operatorname{s}^{-\lim _{\tau \rightarrow+0}}\left(I+M_{\gamma}(\tau)\right)^{-1}=((2+\gamma) I+C)^{-1}$ for $\gamma \in[0,1]$, by Lemma 4.4 we get that $\mathrm{w}-\lim _{\tau \rightarrow+0}(I+\gamma T(\tau))^{-1}$ exists for $\gamma \in[0,1)$ and is given by

$$
\mathrm{w}-\lim _{\tau \rightarrow+0}(I+\gamma T(\tau))^{-1}=\frac{2 I+C}{(2+\gamma) I+C}, \quad \gamma \in[0,1) .
$$

Hence we get

$$
\text { w- } \lim _{\tau \rightarrow+0}(\nu I+T(\tau))^{-1}=\frac{2 I+C}{I+\nu(2 I+C)}=\left(\nu I+(2 I+C)^{-1}\right)^{-1}, \quad \nu \in(1, \infty) .
$$

However, in standard manner we obtain from this relation

$$
\text { S- } \lim _{\tau \rightarrow+0}(\nu I+T(\tau))^{-1}=\left(\nu I+(2 I+C)^{-1}\right)^{-1}, \quad \nu \in(1, \infty),
$$

which immediately implies (4.25).

Finally, for technical reasons we need the following lemma. First we recall that a bounded operator $X$ is called accretive, if $\Re(X h, h) \geq 0$ for any $h \in \mathfrak{H}$.

Lemma 4.6. Let $\{X(\tau)\}_{\tau>0}$ be a sequence of bounded accretive operators on $\mathfrak{H}$. If there is self-adjoint operator $Y$ such that

$$
\mathrm{w}-\lim _{\tau \rightarrow+0}(X(\tau)-\xi)^{-1}=(i Y-\xi)^{-1}
$$


for some $\Re \mathrm{e}(\xi) \leq 0$, then

$$
\text { s- } \lim _{\tau \rightarrow+0}(X(\tau)-\xi)^{-1}=(i Y-\xi)^{-1} .
$$

Proof. We set

$$
W(\tau):=(X(\tau)+\bar{\xi})(X(\tau)-\xi)^{-1} \quad \text { and } \quad W:=(i Y+\bar{\xi})(i Y-\xi)^{-1},
$$

$\tau>0$. One easily verifies that $\{W(\tau)\}_{\tau>0}$ is a family of contractions. Obviously, we have $\mathrm{w}-\lim _{\tau \rightarrow+0} W(\tau)=W$. By

$$
\|W(\tau) h-W h\|^{2}=\|W(\tau) h\|^{2}+\|h\|^{2}-2 \Re \mathrm{e}((W(\tau) h, W h)), \quad \tau>0,
$$

we find

$$
\limsup _{\tau \rightarrow+0}\|W(\tau) h-W h\|^{2} \leq 2\|h\|^{2}-2 \lim _{\tau \rightarrow+0}(W(\tau) h, W h)=0,
$$

which completes the proof.

Theorem 4.7. Let $A$ and $B$ be non-negative self-adjoint operators such that the intersection $\operatorname{dom}\left(A^{1 / 2}\right) \cap \operatorname{dom}\left(B^{1 / 2}\right)$ is dense in $\mathfrak{H}$. If $\phi$ and $\psi$ admissible functions such that the conditions (4.17) are satisfied, then (1.13) holds uniformly for $t \in$ $[0, T]$ and $T>0$.

Proof. Taking into account the representation

$$
\frac{1}{\sqrt{I+M_{0}(\tau)}}(i I+T(\tau))^{-1} \frac{1}{\sqrt{I+M_{0}(\tau)}}=(i I+(1+i) \varphi(\tau A)+K(\tau))^{-1}
$$

we find

$$
\frac{1}{\sqrt{I+M_{0}(\tau)}}(i I+T(\tau))^{-1} \frac{1}{\sqrt{I+M_{0}(\tau)}}=\sqrt{\widetilde{\phi}(\tau A)}\left(Z(\tau)-\xi_{0}\right)^{-1} \sqrt{\widetilde{\phi}(\tau A)}
$$

for $\tau>0$, where $\widetilde{\phi}(y):=\frac{1}{\varphi(y)}, y \in \mathbb{R}_{+}, \xi_{0}=-(1+i)$,

$$
Z(\tau):=i \widetilde{\phi}(\tau A)+\widetilde{S}_{\tau}, \quad \tau>0,
$$

and

$$
\widetilde{S}_{\tau}:=\sqrt{\widetilde{\phi}(\tau A)} K(\tau) \sqrt{\widetilde{\phi}(\tau A)}, \quad \tau>0 .
$$

A straightforward computation shows that

$$
\widetilde{S}_{\tau}=E_{\tau}\left(\frac{I-\phi(\tau A)}{\tau}+\sqrt{\phi(\tau A)} \frac{I-\psi(\tau B)}{\tau}\right) E_{\tau}=E_{\tau} S_{\tau} E_{\tau} \tau>0,
$$

where

$$
S_{\tau}:=\frac{I-F(\tau)}{\tau}, \quad \tau>0,
$$

and $F(\tau):=\sqrt{\phi(\tau A)} \psi(\tau B) \sqrt{\phi(\tau A)}, \tau>0$. Since for each $\tau>0$ the operators $S_{\tau}$ are accretive and $\Re \mathrm{e}(\widetilde{\phi}(\tau A)) \geq 0, \tau \geq 0$, the operator $Z(\tau)$ is accretive and the inverse operator $\left(Z(\tau)-\xi_{0}\right)^{-1}$ exists and its norm is bounded by one for $\tau>0$. From the representation (4.26), Lemma 4.4 and Lemma 4.5 we get

$$
\text { w- } \lim _{\tau \rightarrow+0}\left(Z(\tau)-\xi_{0}\right)^{-1}=(i C-\xi)^{-1}, \quad \xi=-1-2 i,
$$




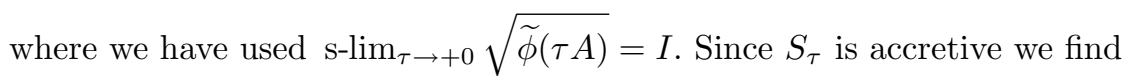

$$
\left(i I+\widetilde{S}_{\tau}-\xi_{0}\right)^{-1}-\left(Z(\tau)-\xi_{0}\right)^{-1}=i\left(i I+\widetilde{S}_{\tau}-\xi_{0}\right)^{-1}(\widetilde{\phi}(\tau A)-I)\left(Z(\tau)-\xi_{0}\right)^{-1}
$$

for $\tau>0$. From (4.28) and (4.29) we get

$$
\mathrm{W}-\lim _{\tau \rightarrow+0}\left(\widetilde{S}_{\tau}-\xi\right)^{-1}=(i C-\xi)^{-1} \text {. }
$$

Applying Lemma 4.6 we find

$$
\text { S- } \lim _{\tau \rightarrow+0}\left(\widetilde{S}_{\tau}-\xi\right)^{-1}=(i C-\xi)^{-1}
$$

which yields

$$
\text { S- } \lim _{\tau \rightarrow+0}\left(\mu I+\widetilde{S}_{\tau}\right)^{-1}=(\mu I+i C)^{-1}, \quad \mu>0
$$

Since

$$
S_{\tau}=-\frac{1}{\tau}\left(I-E_{\tau}\right)+\widetilde{S}_{\tau}, \quad \tau>0
$$

we have

$$
\begin{aligned}
& \left(\mu I+S_{\tau}\right)^{-1}-\left(\mu I+\widetilde{S}_{\tau}\right)^{-1}= \\
& \quad\left(\mu I+S_{\tau}\right)^{-1}\left(\widetilde{S}_{\tau}-S_{\tau}\right)\left(\mu I+\widetilde{S}_{\tau}\right)^{-1}=\frac{1}{\mu \tau}\left(\mu I+S_{\tau}\right)^{-1}\left(I-E_{\tau}\right), \quad \tau>0 .
\end{aligned}
$$

Let $\Delta=[0, d), d>0$, then we have

$$
\left(\mu I+S_{\tau}\right)^{-1} E_{A}(\Delta) h-\left(\mu I+\widetilde{S}_{\tau}\right)^{-1} E_{A}(\Delta) h=\frac{1}{\mu \tau}\left(\mu I+S_{\tau}\right)^{-1}\left(I-E_{\tau}\right) E_{A}(\Delta) h
$$

for $\tau>0$. Since $\left(I-E_{\tau}\right) E_{A}(\Delta) h=0$ if $\tau$ is sufficiently small we find from (4.30) that

$$
\text { S- } \lim _{\tau \rightarrow+0}\left(\mu I+S_{\tau}\right)^{-1}=(\mu I+i C)^{-1}, \quad \mu>0 .
$$

From [2] we get $\operatorname{s-lim}_{n \rightarrow \infty} F(t / n)^{n}=e^{-i t C}$ uniformly in $t \in[0, T], T>0$, which completes the proof.

Corollary 4.8 ([18]). Let $A$ and $B$ be non-negative self-adjoint operators such that $\operatorname{dom}\left(A^{1 / 2}\right) \cap \operatorname{dom}\left(B^{1 / 2}\right)$ is dense in $\mathfrak{H}$. If $\phi(y)=\psi(y)=(1+i y)^{-1}, y \in \mathbb{R}_{+}$, then

$$
\text { s- } \lim _{n \rightarrow \infty}\left((I+i t A / n)^{-1}(I+i t B / n)^{-1}\right)^{n}=e^{-i t C}
$$

uniformly in $t \in[0, T], T>0$.

Proof. One easily verifies that $\phi(\cdot)$ and $\psi(\cdot)$ are admissible functions. Moreover, one has

$$
\phi_{\mathcal{R}}(y)=\psi_{\mathcal{R}}(y)=\frac{1}{1+y^{2}} \geq 0 \quad \text { and } \quad \phi_{\mathcal{I}}(y)=\psi_{\mathcal{I}}(y)=-\frac{y}{1+y^{2}} \leq 0
$$

which shows that the assumptions (4.17) are satisfied. Applying Theorem 4.7 we arrive at the sought conclusion. 
The function $\phi(y)=e^{-i y}, y \in \mathbb{R}_{+}$, does not satisfy the conditions $\Re$ e $(\phi(y)) \geq$ 0 and $\Im \mathrm{m}(\phi(y)) \leq 0$. However, its modification $\phi(y):=e^{-i y} \chi_{[0, \pi / 2]}(y), y \in \mathbb{R}_{+}$, obeys $\Re$ e $(\phi(y)) \geq 0$ and $\Im \mathrm{m}(\phi(y)) \leq 0, y \in \mathbb{R}_{+}$. In particular, the function (1.7) satisfies the conditions $\Re \mathrm{e}(f(y)) \geq 0$ and $\Im \mathrm{m}(f(y)) \leq 0$. The last observation leads to the following claim.

Corollary 4.9. Let $A$ and $B$ non-negative self-adjoint operators in a separable Hilbert space $\mathfrak{H}$ such that $\operatorname{dom}\left(A^{1 / 2}\right) \cap \operatorname{dom}\left(B^{1 / 2}\right)$ is dense in $\mathfrak{H}$. Let $\phi$ and $\psi$ be admissible functions. Then there are real numbers $\delta_{\phi}>0$ and $\delta_{\psi}>0$ such that

$$
\text { s- } \lim _{n \rightarrow+\infty}\left(\phi ( t A / n ) E _ { A } \left(\left(\left[0, n \delta_{\phi} / t\right]\right) \psi(t B / n) E_{B}\left(\left(\left[0, n \delta_{\psi} / t\right]\right)\right)^{n}=e^{-i t C}\right.\right.
$$

holds uniformly in $t \in[0, T], T>0$.

Proof. If the function $\phi$ is admissible, then $\phi(0):=\lim _{y \rightarrow+0} \phi(y)=1$ and $\phi^{\prime}(0)=$ $\lim _{y \rightarrow+0} \frac{\phi(y)-1}{y}=-i$. In particular, this yields $\Re \mathrm{e}(\phi(0))=\lim _{y \rightarrow+0} \phi_{\mathcal{R}}(y)=1$ and $\Im \mathrm{m}(\phi(0))=\lim _{y \rightarrow+0} \phi_{\mathcal{I}}(y)=0$ as well as $\Re \mathrm{e}\left(\phi^{\prime}(0)\right)=\lim _{y \rightarrow+0} \frac{\phi_{\mathcal{R}}(y)-1}{y}=0$ and $\Im \mathrm{m}\left(\phi^{\prime}(0)\right)=\lim _{y \rightarrow+0} \frac{\phi_{\mathcal{I}}(y)}{y}=-1$ where $\phi_{\mathcal{R}}:=\Re \mathrm{R}\left(\phi(y)\right.$ and $\phi_{\mathcal{I}}:=\Im \mathrm{m}(\phi(y))$, $y \in \mathbb{R}_{+}$. Hence there is a $\delta_{\phi}>0$ such that $\phi_{\mathcal{R}}(y) \geq 0$ and $\phi_{\mathcal{I}}(y) \leq 0$ for $y \in\left[0, \delta_{\phi}\right]$, and consequently, the function $\phi(y) \chi_{\left[0, \delta_{\phi}\right]}(y)$ satisfies the assumptions of Theorem 4.7. Similar considerations are valid for $\psi$.

Corollary 4.9 shows that the modified Trotter product formula (1.14) mentioned in the introduction is valid.

\section{Concluding remarks}

To conclude the paper let us list some open problems related to the Trotter-Kato product formula:

(i) The relation between holomorphic Kato and admissible functions is an open question. Of course, the class of admissible functions is (in some sense) larger than the class of holomorphic Kato functions, even if the conditions (4.17) are satisfied. This follows from the fact that far from zero an admissible function can be chosen arbitrarily, in particular, one can extend it by zero. However, a holomorphic Kato function, which is zero on a set of positive Lebesgue measure, equals zero identically. On the other hand, it is not clear whether a holomorphic Kato function satisfies the conditions of admissible functions at zero, cf. Definition 4.1 .

(ii) Is it possible to verify the Trotter-Kato product formula (1.13) for admissible functions if one strengthens slightly the hypotheses made in Section 4, for instance, supposing that $\operatorname{dom}\left(B^{1 / 2}\right) \subseteq \operatorname{dom}\left(A^{1 / 2}\right)$ ?

(iii) Are there non-negative self-adjoint operators $A$ and $B$ such that the Trotter-Kato product formula (1.13) does not hold for a pair of holomorphic Kato functions $\phi$ and $\psi$ ? 
(iv) What can be said about the operator norm convergence of the TrotterKato product formula (1.13)? It is known that for the real time there are several conditions, which guarantee the operator norm convergence, see $[12,19]$ and references therein. For imaginary times, however, the available results are rather restricted, see [11].

\section{Acknowledgment}

The authors are grateful for the hospitality they enjoyed, P.E. in WIAS and H.N. in Doppler Institute, during the time when the work was done. The research was supported by the Czech Ministry of Education, Youth and Sports within the project LC06002. Further we wish to thank Takashi Ichinose who draw our attention to the paper [10].

\section{References}

[1] Vincent Cachia. On a product formula for unitary groups. Bull. London Math. Soc., 37(4):621-626, 2005.

[2] Paul R. Chernoff. Note on product formulas for operator semigroups. J. Functional Analysis, 2:238-242, 1968.

[3] Paul R. Chernoff. Product formulas, nonlinear semigroups, and addition of unbounded operators. American Mathematical Society, Providence, R. I., 1974. Memoirs of the American Mathematical Society, No. 140.

[4] Pavel Exner. Open quantum systems and Feynman integrals. Fundamental Theories of Physics. D. Reidel Publishing Co., Dordrecht, 1985.

[5] Pavel Exner and Takashi Ichinose. A product formula related to quantum Zeno dynamics. Ann. Henri Poincaré, 6(2):195-215, 2005.

[6] Pavel Exner, Takashi Ichinose, Hagen Neidhardt, and Valentin A. Zagrebnov. Zeno product formula revisited. Integral Equations Operator Theory, 57(1):67-81, 2007.

[7] Pavel Exner and Hagen Neidhardt. Trotter-Kato product formula for unitary groups. arXiv:0907.1199v1 [math-ph], 2009.

[8] Paolo Facchi and Saverino Pascazio. Quantum Zeno dynamics: mathematical and physical aspects. J. Phys. A, 41(49):493001, 45, 2008.

[9] William G. Faris. The product formula for semigroups defined by Friedrichs extensions. Pacific J. Math., 22:47-70, 1967.

[10] Takashi Ichinose. A product formula and its application to the Schrödinger equation. Publ. Res. Inst. Math. Sci., 16(2):585-600, 1980.

[11] Takashi Ichinose and Hideo Tamura. Note on the norm convergence of the unitary Trotter product formula. Lett. Math. Phys., 70(1):65-81, 2004.

[12] Takashi Ichinose, Hideo Tamura, Hiroshi Tamura, and Valentin A. Zagrebnov. Note on the paper: "The norm convergence of the Trotter-Kato product formula with error bound" by T. Ichinose and H. Tamura [Comm. Math. Phys. 217 (2001), no. 3, 489-502; MR1822104 (2002e:47048a)]. Comm. Math. Phys., 221(3):499-510, 2001. 
[13] Gerald W. Johnson and Michel L. Lapidus. The Feynman integral and Feynman's operational calculus. Oxford Mathematical Monographs. The Clarendon Press Oxford University Press, New York, 2000. Oxford Science Publications.

[14] Tosio Kato. Trotter's product formula for an arbitrary pair of self-adjoint contraction semigroups. In Topics in functional analysis (essays dedicated to M. G. Krein on the occasion of his 70th birthday), volume 3 of Adv. in Math. Suppl. Stud., pages 185-195. Academic Press, New York, 1978.

[15] Michel Lapidus. Formules de moyenne et de produit pour les résolvantes imaginaires d'opérateurs auto-adjoints. C. R. Acad. Sci. Paris Sér. A-B, 291(7):A451-A454, 1980.

[16] Michel L. Lapidus. Generalization of the Trotter-Lie formula. Integral Equations Operator Theory, 4(3):366-415, 1981.

[17] Michel L. Lapidus. The problem of the Trotter-Lie formula for unitary groups of operators. Séminaire Choquet: Initiation à l'Analyse. Publ. Math. Univ. Pierre et Marie Curie (Paris IV). 20 ème année, 1980/81, 46:1701-1746, 1982.

[18] Michel L. Lapidus. Product formula for imaginary resolvents with application to a modified Feynman integral. J. Funct. Anal., 63(3):261-275, 1985.

[19] Hagen Neidhardt and Valentin A. Zagrebnov. Trotter-Kato product formula and operator-norm convergence. Comm. Math. Phys., 205(1):129-159, 1999.

[20] Edward Nelson. Feynman integrals and the Schrödinger equation. J. Mathematical Phys., 5:332-343, 1964.

[21] Béla Sz.-Nagy and Ciprian Foias. Harmonic analysis of operators on Hilbert space. Translated from the French and revised. North-Holland Publishing Co., Amsterdam, 1970.

[22] Hale F. Trotter. On the product of semi-groups of operators. Proc. Amer. Math. Soc., 10:545-551, 1959.

Pavel Exner

Department of Theoretical Physics, NPI, Academy of Sciences, CZ-25068 Řež and Doppler Institute, Czech Technical University, Břehová 7, CZ-11519 Prague, Czech Republic e-mail: exner@ujf.cas.cz

Hagen Neidhardt

Weierstrass Institute for Applied Analysis and Stochastics, Mohrenstrasse 39, D-10117 Berlin, Germany

e-mail: neidhard@wias-berlin.de

Valentin A. Zagrebnov

Université de la Méditerranée (Aix-Marseille II) and Centre de Physique Théorique, Luminy-Case 907, 13288 Marseille Cedex 9, France

e-mail: zagrebnov@cpt.univ-mrs.fr 